\title{
AN EXAMINATION OF EMOTION REGULATION STRATEGY USE ON RECOGNITION MEMORY
}

by

\author{
Beverley Fredborg \\ B.Sc. (Honours), University of Winnipeg, 2015
}

\author{
A thesis presented to Ryerson University \\ in partial fulfillment of the \\ requirements for the degree of \\ Master of Arts \\ in the Program of \\ Psychology \\ Toronto, Ontario, Canada, 2018 \\ CBeverley Fredborg 2018
}




\section{Author's Declaration}

I hereby declare that I am the sole author of this thesis. This is a true copy of the thesis, including any required final revisions, as accepted by my examiners.

I authorize Ryerson University to lend this thesis to other institutions or individuals for the purpose of scholarly research.

I further authorize Ryerson University to reproduce this thesis by photocopying or by other

means, in total or in part, at the request of other institutions or individuals for the purpose of scholarly research.

I understand that my thesis may be made electronically available to the public. 
An Examination of Emotion Regulation Strategy Use on Recognition Memory

\author{
(Master of Arts, 2018) \\ Beverley K. Fredborg \\ Psychology \\ Ryerson University
}

\begin{abstract}
Emotion regulation (ER) refers to the use of various strategies to modify an emotional response and has important implications for memory of emotional events. During this study, participants were instructed to first enhance, maintain, or suppress their emotional responses while viewing unpleasant and neutral images and then report the ER strategies they used during the task. A surprise memory test for these images known as the Remember/Know procedure was then conducted immediately after encoding and following a one-week delay. Overall, negative images were better remembered than neutral images. Moreover, images paired with the instruction to enhance one's emotional responses were better remembered than images paired with the instruction to maintain or suppress, on the first test day only. Specific types of ER strategies used were not reliably associated with memory for emotional images. This research is the first to inform of the impact of spontaneous ER strategy use on memory.
\end{abstract}




\section{Acknowledgements}

I am deeply grateful for several people who have been instrumental in assisting me in this endeavour. Foremost is my research supervisor, Dr. Todd Girard, whose support and mentorship has been incredibly valuable during the thesis process and beyond. Dr. Girard's tireless dedication to his students is remarkable and has been of great benefit to me throughout these past two years. Importantly, I am grateful for the mentorship of Dr. Julia Spaniol, who has graciously offered insightful comments on this thesis. These insights have furthered my understanding of the literature and have encouraged me to examine my work through a more critical lens. I also thank my thesis committee members, Dr. Lixia Yang and Dr. Frank Russo, for offering their time in supporting me at the end stages of the thesis process. Lastly, I am grateful for the support of Justice Cupid, who assisted on data collection for this thesis with enthusiasm and good cheer.

I am acutely aware of how fortunate I am to have had the opportunities I have had in my life. As such, I would like to offer words of gratitude to the individuals who have shaped me as a budding clinician-scientist. It would be remiss of me to not firstly acknowledge the 2016-2018 clinical psychology faculty, as well as the graduate student cohort at Ryerson, who have taught me more than I ever thought possible throughout these past two years. In this same vein, I must thank my undergraduate thesis supervisor, Dr. Steve Smith, who provided me with myriad opportunities to grow as a researcher. Third, I would like to acknowledge the invaluable and often unacknowledged contributions to my learning made by my lab-mates, coworkers, friends, and family. Thank you for everything. I especially want to thank my partner, Kevin, for both his technical support on this project and his support in us moving from Winnipeg to Toronto to pursue higher education. His uncanny ability to simultaneously be a peer-editor, mentor, confidant, role-model, and decent human being is unparalleled. 


\section{Contents}

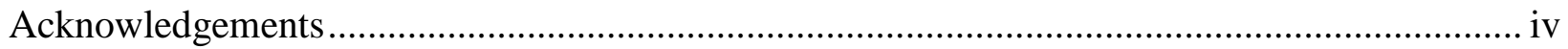

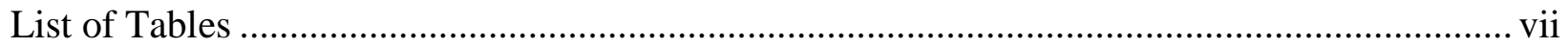

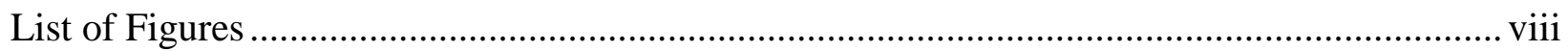

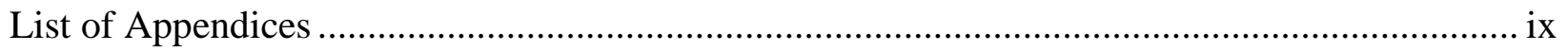

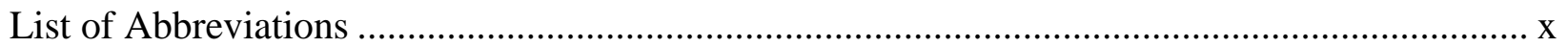

An Examination of Emotion Regulation on Recognition Memory ......................................... 1

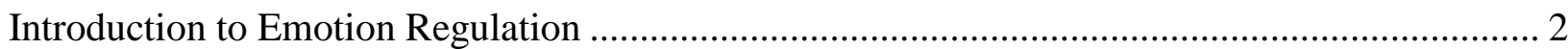

Current Challenges in the Emotion Regulation and Memory Literature ................................ 5

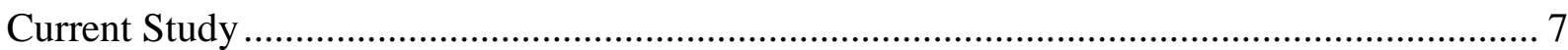

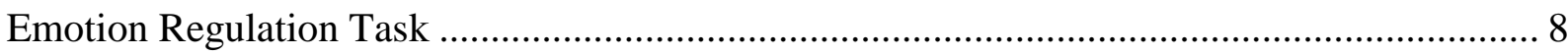

Emotion Regulation Task Questionnaire ................................................................... 9

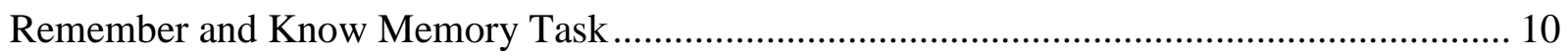

Rationale for examining RK judgments. ...................................................................... 13

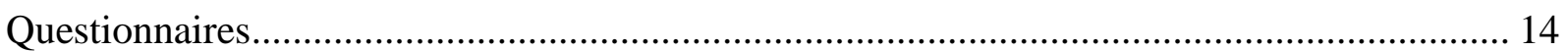

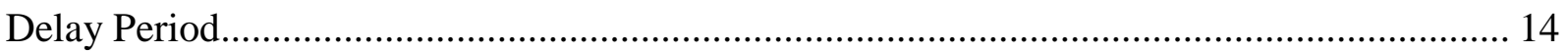

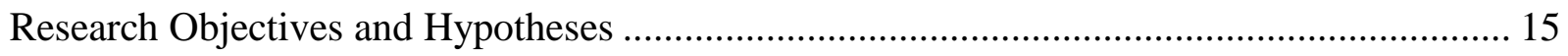

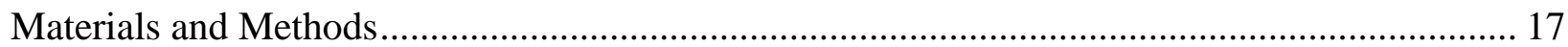

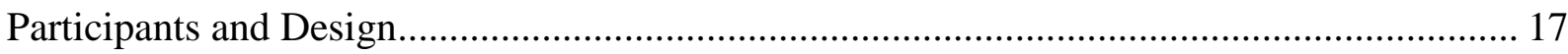

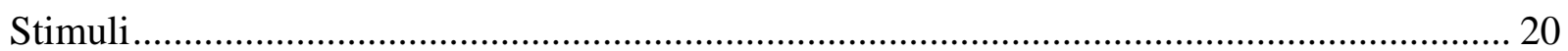

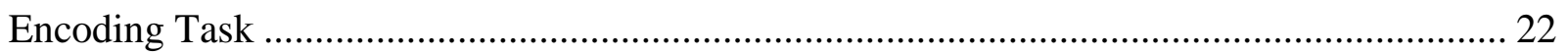

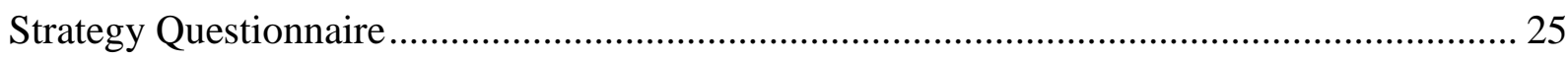

Distraction Period Tasks ............................................................................................... 26

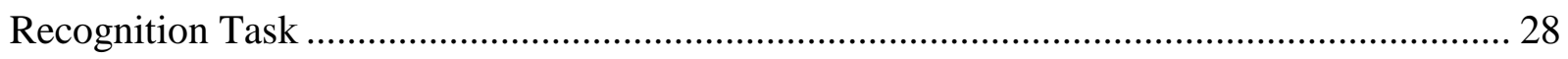

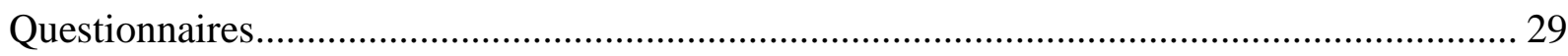

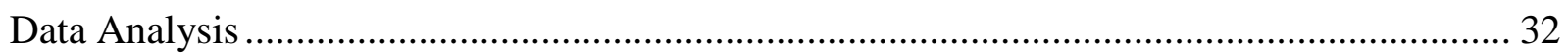

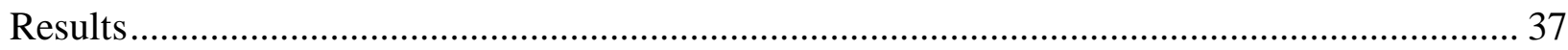

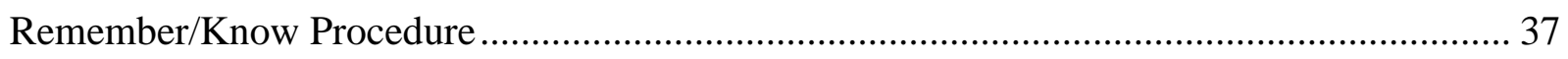

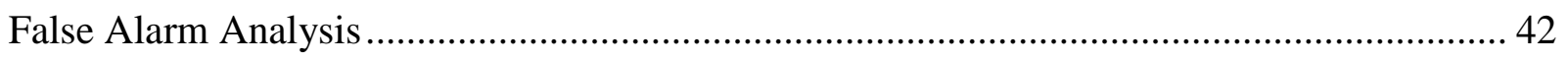

Emotion Regulation Strategy Use.............................................................................. 42

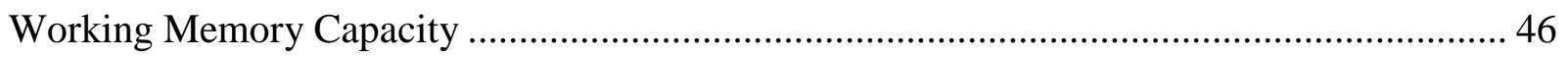

Correlations Between Emotion Regulation Strategy Use and Questionnaire Data ................ 47

Correlations between Emotion Regulation Strategy Use and Memory Outcomes .................. 48

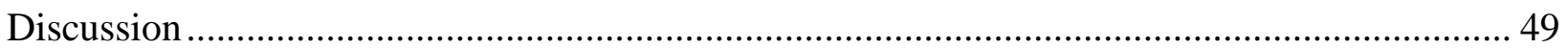


Emotional Enhancement of Memory …………………...................................................... 50

Influence of Emotion Regulation Task Instructions on Memory ............................................ 51

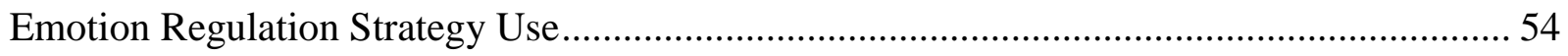

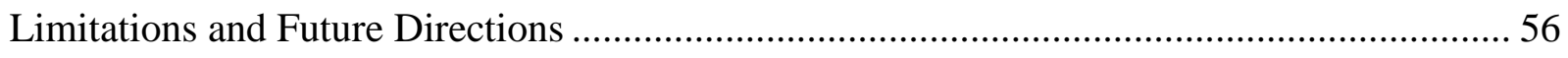

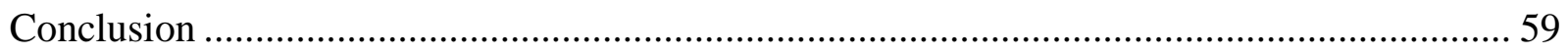

Appendix A: Demographic and Screening Interview (self-created, 2017)…………................... 60

Appendix B: Emotion Regulation Verbal Instructions Administered during the Encoding Phase

Appendix C: Emotion Regutan 6

Appendix D: Computerized Instructions for the Remember/Know Task (Adapted from Patel,

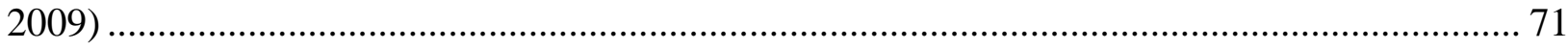

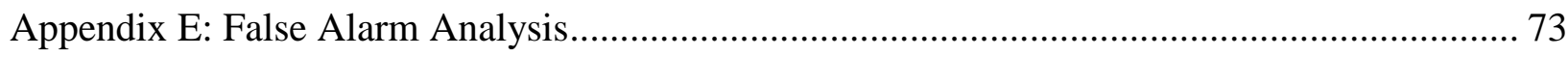

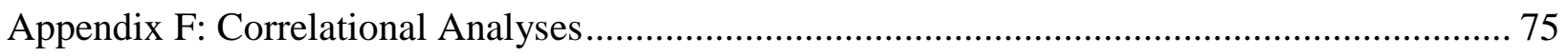

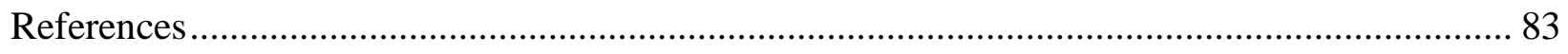




\section{List of Tables}

Table 1. Emotion Regulation Strategy Use During Block 1 and Totalled Across Blocks of the

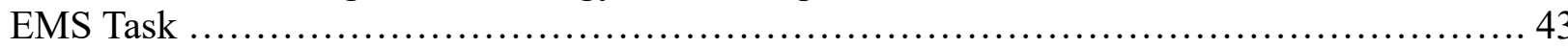

Table 2. Number of Emotion Regulation Strategies Used During Block 1 of EMS Task and

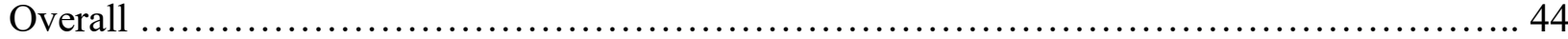




\section{List of Figures}

Figure 1. Exemplar unpleasant and neutral photos from the OASIS (Kurdi et al., 2017) ...... 21

Figure 2. The emotion regulation paradigm adapted from Jackson and colleagues (2000) ..... 24

Figure 3. Mean hit rate values for correct remember and know responses, for both negative and

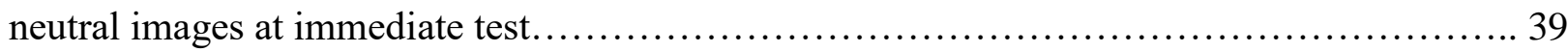

Figure 4. Mean hit rate values for correct remember and know responses, for the enhance,

maintain, and suppress instructions during the EMS task at immediate test $\ldots \ldots \ldots \ldots \ldots \ldots . . . .40$

Figure 5. Mean hit rate values for correct remember and know responses, for both negative and neutral images after the one-week delay period 


\section{List of Appendices}

Appendix A: Demographic and Screening Interview.....................................60

Appendix B: Emotion Regulation Verbal Instructions Administered during the Encoding

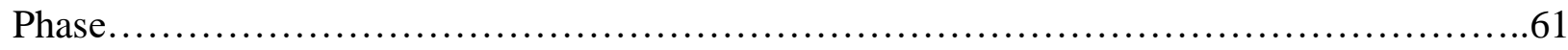

Appendix C: Emotion Regulation Strategy Questionnaire...................................62

Appendix D: Computerized Instructions for the Remember/Know

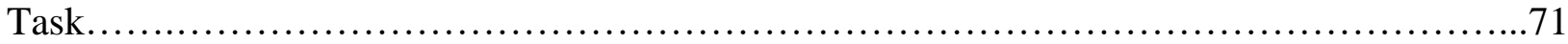

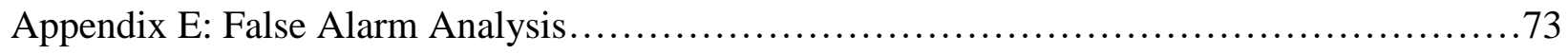

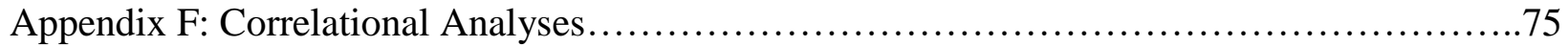


ANOVA: Analysis of variance

\section{List of Abbreviations}

$\mathrm{B}_{\mathrm{r}}$ : Response bias

EEM: Emotional enhancement of memory

EMS: Enhance/Maintain/Suppress

FA: False alarm

M: Mean

OASIS: Open Affective Standardized Image Set

$\mathrm{P}_{\mathrm{r}}$ : Discrimination index

RK: Remember/Know

SD: Standard deviation

SEM: Standard error of the mean

WMC: Working memory capacit 


\section{An Examination of Emotion Regulation on Recognition Memory}

Emotion regulation refers to the collection of conscious and nonconscious strategies we use to modify our emotions (Gross, 1999). Emotion regulation affects not only how we understand and navigate our complex emotional environment, but also our memory for emotional events. Although the literature on emotional memory has established that specific emotion regulation strategies differentially affect memory for emotional events (as will be reviewed below; Gross, 2001), no studies have explored the relationship between the spontaneous use of emotion regulation strategies and memory. Therefore, this thesis uniquely contributes to the literature in two distinct ways: It assesses the effect of spontaneous emotion regulation strategy use on recognition memory, and further decomposes this form of memory into responses that differentially rely on recollection and familiarity processes. To examine this relationship, an emotion regulation task was combined with a test of recognition memory. To further examine the temporal effects of emotion regulation on memory for emotional images, the memory test was administered both after a short delay (15 minute), as well as after a 1-week delay. Furthermore, the participants' use of emotion regulation strategies outside of the laboratory, as well as state levels of depression, anxiety, and stress, were assessed to both characterize the sample and to explore the relationship between emotion regulation strategy use in daily life and in the laboratory environment.

Before describing this experiment in more detail, this thesis will first introduce emotion regulation to the reader and provide a brief overview of the emotional memory literature. Following this review, a discussion of the methodologies employed in the experiment will be introduced, followed by the results of the experiment. Lastly, a discussion of the experimental findings will be presented, including an overview of this study's limitations. Future directions will also be discussed. 


\section{Introduction to Emotion Regulation}

Emotions are the filter through which we view the world. In our daily lives, emotions such as sadness, joy, guilt, and fear alter our thoughts, physiology, and behaviour, and enhance our ability to survive and, in most instances, thrive (Gross, 2001; Sheppes et al., 2014). As such, the ability to regulate our emotions - to consciously or non-consciously determine which emotions should be attended to and altered - is paramount to our day-to-day wellbeing (Bonanno, Papa, Lalande, Westphal, \& Coifman, 2004).

Researchers have defined the ability to modify our responses to emotional events as emotion regulation (Gross, 1999). More specifically, emotion regulation is the process by which we use a varied collection of conscious and nonconscious strategies to alter our emotions; for example, to enhance or decrease our emotional experience, or to change our emotional experience into one that is more pleasant (Gross, 1999). Several studies have found that the flexible use of these strategies is vital to the effective navigation of one's complex emotional environment (e.g., Bonanno et al., 2004; Sheppes et al., 2014). For instance, when struggling to decrease anger, an individual may have difficulties in regulating his behaviour while he is angry, leading to potential harm to himself or others.

There are two broadly defined categories of strategies for emotion regulation (Gross, 2001). Antecedent-focused strategies, such as cognitive reappraisal, aim to modulate the meaning of an emotional event prior to responding in a specific way to that emotion. For instance, an individual may watch a movie depicting gruesome violence or torture, and feel overwhelmingly disgusted or frightened by the images. However, if that individual was engaging in cognitive reappraisal, he may focus on the fact that the movie is a Hollywood film, rather than a documentary. In this way, cognitions about the movie alter his emotional experiences, 
increasing his enjoyment of the film. In contrast, response-focused strategies, such as enhancement or suppression, aim to modulate the emotional response during the emotiongenerative process. For instance, an individual watching the same movie may try to suppress her emotional responses by altering her facial expression to a more neutral pose, or by slowing her breathing to reduce her heart rate. Although both types of strategies may result in decreased emotional responses to stimuli, they are deployed at different time points throughout the emotion-generative process (Gross, 2001).

Although the ability to flexibly deploy emotion regulation strategies such as cognitive reappraisal and suppression is a skill most healthy individuals are adept at using, impairments in emotion regulation are pronounced in individuals with several forms of mental illness (Ochsner $\&$ Gross, 2005). For example, for individuals with borderline personality disorder, the ability to regulate negative emotions may be a skill that is consciously practiced and learned through therapy, rather than modelled by significant others or learned through life experience (Linehan, 1993). Furthermore, for individuals who have experienced a traumatic event, the ability to regulate emotions may be a protective factor that negatively predicts the development of posttraumatic stress disorder (PTSD; New et al., 2009). Thus, the study of how and why individuals use specific emotion regulation strategies in various contexts, and what strategies are most effective on an individual basis, is especially important for developing treatment for disorders associated with emotional dysregulation. Despite its importance, little research has been published on how individuals choose (whether consciously or unconsciously) which strategies to use in specific contexts (Sheppes et al., 2014), and how these choices affect memory for the emotional event. 
A recent conceptual framework of emotion regulation strategy choice has been presented by Sheppes and colleagues (2014). This framework draws from both information processing literature and the process model of emotion regulation outlined by Gross and Thompson (2007). Sheppes and colleagues (2014) suggest that our limited cognitive capacity results in competition between emotion generation and the emotion regulation process itself for dominance over behaviour. In this framework, emotion regulation occurs throughout two distinct cognitive stages: an attentional selection stage, in which emotional situations are either attended to or disengaged from, and a semantic meaning-processing stage, during which time the meaning of the emotional situation is processed and potentially manipulated or elaborated upon. In the first stage, an individual may distract herself from the emotional event, such that interfering neutral thoughts are generated before the event is represented in working memory. Once the emotional information has bypassed the attentional stage and has entered working memory, cognitive reappraisal may occur. During this process, the emotional information is manipulated such that a neutral reinterpretation of its meaning may be established. This new meaning conflicts with the event's original emotional significance and thus affects her behaviour, and, subsequently, her memory for the event.

The processes that occur during the two stages of emotional processing result in a costbenefit trade-off, to the effect that blocking high-intensity emotional information during the attention selection stage may be immediately emotionally beneficial (i.e., the emotional intensity is lessened), but may lead to poor memory outcomes due to the lack of deeper evaluation and processing (Sheppes et al., 2014). For instance, an individual who blocks high-intensity emotional information may similarly block key details that occur during the emotional event, such as what a family member said to him during a particularly emotional argument. On the 
other hand, if the emotional information does bypass the attention selection stage, cognitive reappraisal also results in similar cost-benefit trade-offs. By processing emotional information more deeply, he may be vulnerable to unpleasant high-intensity emotional information, such as guilt about the content of the family argument. However, he will be better able to process, evaluate, and remember the information later, which is adaptive. For instance, it may be beneficial for him to more deeply process and remember the details of the argument, as it could help him learn how to avoid arguments with the family member in the future.

\section{Current Challenges in the Emotion Regulation and Memory Literature}

Not only is the study of emotion regulation vital for informing treatment of mental disorders characterized by difficulties in emotion regulation, but it is also valuable in the study of memory, as the ability to recollect emotional events is impacted by the strategy used at the time of encoding (Gross, 2001). For instance, one consistent finding is that consciously replacing one's negative facial expression with a neutral expression (“expressive suppression”) during encoding of emotional images leads to worse explicit memory for those images (Dillon, Ritchey, Johnson, \& LaBar, 2007; Richards \& Gross, 1999). Furthermore, engaging in cognitive reappraisal during encoding leads to better memory for emotional images than suppression (e.g., Dillon et al., 2007).

The literature is complicated, however, by the use of different definitions of emotion regulation strategies. While several studies instruct participants to "cognitively reappraise" a situation depicted in an emotional image, the way this has been operationalized varies between studies. For example, some explain cognitive reappraisal as reimagining a positive outcome of the situation (e.g., Dillon et al., 2007), whereas others instruct participants to cognitively change the emotional meaning of the situation (e.g., New et al., 2009). In their study of the effect of 
emotion regulation strategies on memory, Dillon and colleagues (2007) conducted two experiments in which individuals viewed negative and neutral images and were asked to modulate their emotional responses while viewing them. In the first experiment, participants were instructed to maintain, increase, or decrease the personal relevance of the emotional image while viewing it using mental imagery. In the second experiment, participants were again instructed to either maintain or enhance the personal relevance of the image; however, in place of the "decrease" instruction, participants were instructed to modulate their facial expression such that an outside observer could not guess how they were feeling in response to that image. Both experiments used a surprise free-recall memory task in which individuals were asked to list as many details as possible regarding the images they had viewed during the encoding phase of the experiment. Thus, the researchers could directly compare the effects of cognitive reappraisal (i.e., decreased personal relevance) and expressive suppression (i.e., facial expression manipulation) on memory for the images, as the only difference between the two experiments were the instructions provided to participants for the "decrease" (Experiment 1) or "suppress" (Experiment 2) instruction. In accordance with literature on emotion regulation and memory (e.g., Richards \& Gross, 1999), the results of the study indicated that overall, pictures paired with the instruction to enhance were well-recollected, while pictures paired with the instruction to suppress facial expressions were poorly recollected. In contrast, for pictures paired with the instruction to decrease the personal relevance of the image, memory for the images varied by picture type. Specifically, unpleasant images with this instruction were well-recollected, while the neutral images were poorly recollected during these trials (Dillon et al., 2007). Although in both experiments participants were instructed to decrease their emotional response to emotional 
images either by reappraisal or expressive suppression, the type of instruction and valence of the image differentially affected their ability to recollect the images.

As noted above, studies vary on the instructions provided to participants during experimental tasks related to emotion regulation strategy use. In contrast to Dillon and colleagues (2007), for example, New and colleagues (2009) instructed participants to imagine a less negative ("decrease" instruction) or a more negative ("enhance" instruction) situational outcome for the protagonist depicted in unpleasant images, rather than to manipulate the personal relevance of the image. Therefore, although cognitive reappraisal was the primary emotion regulation strategy investigated in both the studies by New and colleagues (2009) and Dillon and colleagues (2007), the way in which participants cognitively reappraised the study images differed. Such discrepancies hinder the ability to integrate findings in the literature.

An additional limitation of studies using specific instructions for how to regulate emotions is a potential lack of generalizability to real world situations. For example, it is possible that participants would use different emotion regulation strategies in daily life over those they are instructed to use in the laboratory. By allowing participants to freely choose the way they enhance, maintain, or suppress their emotional response to images, the external validity of these procedures may be increased. Furthermore, by including secondary measures such as mindfulness; levels of anxiety, depression and stress; and emotion regulation in daily life, associations between memory performance and emotion regulation strategy use in the laboratory can be further explored.

\section{Current Study}

The current study addresses the issue of external validity in this literature by examining how the spontaneous use of emotion regulation strategies to enhance, maintain, or suppress 
emotional responses relates to memory for both neutral and emotional stimuli. To this end, an emotion regulation task hereby referred to as the Enhance/Maintain/Suppress (EMS) paradigm (Jackson et al., 2000), as well as a test of recollection and familiarity known as the Remember/Know (RK) procedure (Tulving, 1985) was conducted. Participants' use of emotion regulation strategies during the encoding task was also assessed using a novel strategy questionnaire created for this study. Given that these paradigms instruct participants to describe which strategies they used while viewing emotional images, this study examines conscious emotion regulation strategy use that is deployed after an emotional response is generated, rather than nonconscious strategies or strategies deployed earlier in the emotion-generative process (i.e., situation selection, attentional deployment; Gross, 2001). Furthermore, to better characterize the cognitive abilities of the participant sample, shortened versions of three wellestablished tests of working memory, the Reading Span (RSPAN), the Operation span (OSPAN), and the Symmetry Span (SSPAN; Oswald, McAbee, Redick, \& Hambrick, 2015), were administered. Finally, state levels of depression, anxiety, stress, mindfulness, and use of emotion regulation strategies in daily life were assessed using valid and reliable test measures. To this end, the following section will review the background literature on each of these measures, followed with a discussion of the main hypotheses and the experimental procedure.

\section{Emotion Regulation Task}

In congruence with the studies discussed above (e.g., Dillon et al., 2007; New et al., 2009), the EMS paradigm initially developed by Jackson and colleagues (2000) instructs participants to use strategies to suppress, enhance, or maintain their emotional responses toward emotional images. In contrast, however, this seminal study included neither specific instructions on how to regulate the emotions that arose while viewing the images, nor a measure of memory 
outcomes (as in Dillon et al., 2007) or brain activation (as in New et al., 2009) while viewing these emotional images. Instead, participants' eye blink startle magnitude and corrugator muscle activity were measured to characterize their ability to enhance, maintain, or suppress emotional responses to images. Further, to provide insight on which emotion regulation strategies participants were spontaneously using, participants completed three strategy questionnaires throughout the task. The responses to these questionnaires indicate that while instructed to suppress their emotions, $40 \%$ of participants used a cognitive strategy such as focusing on a positive outcome of the situation depicted in the image (i.e., cognitively reappraising the situation seen in the image) or focusing on a positive aspect of the image (i.e., purposefully choosing where to focus their attention, referred to as "attentional deployment"; Gross, 2001). Additionally, 56\% of participants attempted to rationalize the situation depicted in the image, which again suggests a reappraisal-type strategy, though this strategy may not necessarily involve the addition of emotional information (e.g., imagining that a man depicted in an image is walking into a bank to ask for a loan for his business may contain less salient emotional information than imagining that same man is walking into a bank to ask for a loan to support his dying relative). A further $4 \%$ reported idiosyncratic uses of emotion regulation strategies. These responses suggest that cognitive strategies, such as various forms of cognitive reappraisal, may be commonly used when individuals are tasked to regulate their emotions in the laboratory setting.

\section{Emotion Regulation Task Questionnaire}

Participants in this study were hypothesized to use a range of emotion regulation strategies, similar to those reported by participants in the study by Jackson and colleagues (2000). Using this study as a model, the EMS task instructions did not specifically instruct 
participants on which specific strategies to use, but rather guided them to use whichever strategy or strategies they deemed most effective to enhance, maintain, or suppress their emotional responses. However, unlike the study by Jackson and colleagues (2000), this study gathered more nuanced information on participants' use of emotion regulation strategies during the task to both characterize the sample and to elucidate the relationship between emotion regulation and memory. To this end, after each block of 40 images of the EMS task, participants completed a self-created (2017) emotion regulation questionnaire to determine which strategies they had spontaneously used to regulate their emotions while viewing the previous 40 images, per instruction type (i.e., Enhance, Maintain, or Suppress). This questionnaire is described in more detail in the Materials and Methods section below.

Subsequently, memory for the emotional images was assessed using a popular test of recollection and familiarity known as the RK procedure (Tulving, 1985).

\section{Remember and Know Memory Task}

The RK procedure developed by Tulving (1985) is the most widely-used method for measuring recollection- and familiarity-based processes of memory (Migo et al., 2012). In the RK procedure, participants are asked to rate their memory of previously encoded pictures by indicating that they either "remember" (i.e., the individual can vividly recollect previously viewing the image such that he or she is able to recollect details about the viewing experience, such as the thoughts he or she had while encoding), "know" (i.e., the individual has a strong feeling of familiarity with the image, but cannot recollect distinctly the event of viewing it), or have no memory of the image ("new"; Migo et al., 2012). The RK procedure was developed based on Tulving's (1985) model of memory, in which there are two dissociable states of awareness associated with conscious memory retrieval: a) autobiographical retrieval from 
episodic memory (e.g., recollection) and b) retrieval of a memory in which one is aware that they had previously encountered the content of the memory, yet are not aware of the autobiographic context the memory was situated in (e.g., familiarity; Wixted \& Mickes, 2010).

Although researchers have proposed several distinct dual-process theories of recognition memory (e.g., Tulving, 1985; Yonelinas, 2002), one commonality is that recollection and familiarity are related, but separate, processes that are associated with overlapping, but nonetheless dissociable, systems (Yonelinas, 2002). In Tulving's (1985) model, “the episodic system stores personally experienced events and their temporal relations to each other, whereas the semantic system stores general knowledge about the world" (Yonelinas, 2002, p. 446). Thus, in this model, recognition relies on both the episodic and semantic systems of memory, whereas familiarity relies on the semantic system alone (Yonelinas, 2002).

Despite experimental evidence that recollection and familiarity are dissociable processes (see Yonelinas, 2002, for a review), the dual-process theory of recognition is a point of contention in the literature (Dunn, 2008; Wixted \& Mickes, 2010). Indeed, proponents of singleprocess models of recognition memory have critiqued the claim that the RK procedure differentiates between recollection and familiarity; by contrast, they argue that the responses one makes while completing the RK procedure truly represent an individual's confidence in their memory, such that remember responses indicate high memory confidence, while know responses indicate low confidence (Dunn, 2008; Wixted \& Mickes, 2010). Specifically, Wixted and Stretch (2004), among others, have demonstrated experimentally that remember judgments tend to be associated with higher levels of memory strength than know judgments. In other words, this model suggests that when making a RK judgment, individuals "collect evidence" of their 
memory, such that the stronger evidence one has, the more likely they are to make a remember response (Dunn, 2008).

The above-described single process model of memory is based on the unequal-variance signal-detection theory of memory (Dunn, 2008). This theory operates on the assumption that previously encoded stimuli are associated with higher average memory strength and less variability on the memory strength outcome than new stimuli (Wixted \& Mickes, 2010). This theory thus predicts that, when a certain threshold of memory strength is reached by an individual making a RK judgment, a know judgment switches to a remember judgment (Wixted \& Mickes, 2010).

Due to the continued debate on singular- versus dual-process models of recognition memory, researchers have been developing theoretically-derived alternative models of recognition memory. In one such model, Wixted and Mickes (2010) combine unequal-variance signal-detection theory with a dual-process model of recognition memory. Their new model, termed the "continuous dual-process model of recognition memory," first assumes that both recollection and familiarity processes are continuous signal-detection processes (in contrast to other models which posit that recollection is a dichotomous variable, whilst familiarity is continuous), and second, that RK judgments are based on different memory strength dimensions, rather than on the same dimension as posited by several single-process models of recognition (Dunn, 2008; Wixted \& Mickes, 2010). Thus, the model assumes that RK judgments are based on a recollection dimension and a familiarity dimension, respectively, such that, according to this model, individuals are consciously aware of whether their RK judgments were based on recollection or familiarity processes of memory (though these processes are not assumed to be completely independent; Wixted \& Mickes, 2010). 
Rationale for examining RK judgments. As of this writing, no studies have examined how emotion regulation strategies influence recognition memory for emotional images. Indeed, although the studies discussed herein examine the effects of emotion regulation on memory, only free recall (as per Dillon et al., 2007), cued-recall, and cued-recognition (as per Richards \& Gross, 1999) tasks have been employed by researchers. Thus, a specific examination of the effects of emotion regulation strategies on remember and know responses is warranted for a more nuanced understanding of these processes.

Based on research by Dillon and colleagues (2007), it is expected that both the instruction to either enhance, maintain, or suppress emotional responses to images, as well as the strategy used to do so, will differentially influence participants' remember and know responses. Dillon and colleagues (2007) reported that images paired with the instruction to suppress were recalled with similar accuracy as those images paired with the instruction to enhance, but only when participants used the personal relevance strategy to suppress their emotions. This finding supports that emotion regulation strategies differentially affect recollection. Thus, it is expected that comparable results will be reflected by remember responses in the RK paradigm. Less is known, however, about whether these strategies may also influence know responses. In addition, Dillon and colleagues reported that use of autonomic strategies to suppress emotional responses led to poorer recall compared to images that were cognitively reappraised. It may be the case, however, that familiarity for these images remained intact, because it is a semantic, gist-based process that may be more resistant to forgetting than recollection (Yonelinas, 2002). Moreover, these effects may be moderated by the valence of the image viewed, such that this relationship may only be significant for negative images as opposed to neutral. As such, this study examines 
how emotion regulation instruction and further, the strategies used when instructed to regulate negative emotions, may differentially affect remember and know responses.

\section{Questionnaires}

In addition to the strategy questionnaire administered during the EMS task, participants also completed several questionnaires related to the strategies they naturally use to regulate their emotions; their current levels of depression, anxiety, and stress; and their use of mindfulness practices in their daily life. These questionnaires, which included the Emotion Regulation Questionnaire (ERQ; Gross \& John, 2003), the Trait Meta-Mood Scale (TMMS; Salovey, Mayer, Goldman, Turvey, \& Palfai, 1995), Depression Anxiety Stress Scales-21 (DASS-21; Lovibond \& Lovibond, 1995) and the Mindful Attention and Awareness Scale (MAAS; Brown \& Ryan, 2003) indicate: (a) whether the strategies one uses in naturalistic settings are similar to those spontaneously used during the EMS paradigm; (b) whether one's level of experience using specific emotion regulation strategies predicts their ability to correctly assign a remember response to emotional images; and (c) whether one's state levels of depression, anxiety or stress is predictive of both the types of emotion regulation strategies used both in the laboratory and in daily life, as well as one's memory for emotional images.

\section{Delay Period}

Participants completed the experiment over two separate test days, one week apart, to assess both immediate and long-term effects of emotion regulation on recognition memory. Consistent with consolidation/modulation theories, enhanced memory for emotional stimuli beyond that of neutral images (i.e., the emotional enhancement of memory effect [EEM]) may be particular to long delays (i.e., > 24h; Sommer, Gläscher, Moritz, \& Büchel, 2008; Sharot, Verfaellie, \& Yonelinas, 2007; Sharot \& Yonelinas, 2008) and we expected to observe such an 
emotion by delay interaction on memory here. As such, by employing this one-week delay period, comparison of RK scores on the second test day to RK scores on the first test day allows for an assessment of the EEM effect over a longer duration (Ritchey, Dolcos, \& Cabeza, 2008). Finally, questionnaire data for each participant was expected to provide insight on any discrepancies between memory for emotional images on the day of encoding and memory performance after one week.

\section{Research Objectives and Hypotheses}

The main objective of the current study is to examine how the spontaneous use of emotion regulation strategies in the laboratory setting relates to memory for emotional stimuli. Specific hypotheses address the relationship between the EMS task instructions and levels of accuracy and conscious awareness at retrieval on the RK procedure (i.e., remember or know responses for previously encoded items; Tulving, 1985):

1) Because emotional events are typically better remembered than non-emotional events (EEM effect; Sommer et al., 2008), it is expected that emotional images will be better remembered than neutral images, resulting in more instances of remember responses than know responses for negative images as compared to neutral images (i.e., an image valence by RK response interaction).

2) On the second test day, the EEM effect may be larger than on the first test day. This is because the neutral images may be easier to forget over the one-week delay than the negative images (i.e., a test day by valence interaction; Sharot et al., 2007; Sharot \& Yonelinas, 2008; Sommer et al., 2008).

3) In congruence with results reported by Dillon and colleagues (2007), it is expected that the omnibus ANOVA will reveal a main effect of task instructions, such that 
images paired with the enhance instruction will elicit more remember responses than images paired with either the maintain or suppress instructions.

4) The reported percentage use of each emotion-regulation strategy during the EMS task is predicted to influence subsequent memory accuracy.

a. Based on the influence of emotion regulation strategies on recall memory (Dillon et al., 2007; Gross, 2001), participants who tend to use cognitive reappraisal strategies to enhance their emotions will have more accurate remember responses than participants who tend to use autonomic methods (e.g., breathing more deeply, tensing muscles, etc.).

b. Similarly, based on findings by Dillon and colleagues (2007), it is expected that the percentage of time that cognitive reappraisal strategies are used to suppress emotional responses will be positively correlated with the amount of correct remember responses seen for these images. Moreover, these responses will reach similar levels to those of the images paired with the enhance instruction. However, if autonomic or other idiosyncratic strategies are more often used to suppress emotional responses to the images, memory performance will be impaired, and will result in fewer correct remember responses than those images paired with the maintain instruction.

5) Distinct types of cognitive reappraisal strategies will yield differential associations with memory.

a. Participants who tend to increase the personal relevance of the image to enhance the emotions they experience while viewing the image will have better memory accuracy (i.e., the highest remember hit rate and lowest 
remember false alarm rate) than participants who tend to imagine a different scenario or outcome of the scene depicted in the image. This is based on the "self-reference effect", which is the finding that self-referent information is better remembered than other-referent information (Turk, Cunningham, \& Macrae, 2008).

Secondary objectives include: examining which emotion regulation strategies individuals use in their day-to-day life and whether these strategies differ from those used in the laboratory, examining how other individual difference measures (i.e., questionnaire data) relate to both strategy choice and memory for emotional stimuli, and how WMC relates to emotion regulation strategy use and memory outcomes.

\section{Participants and Design}

\section{Materials and Methods}

Seventy-three participants enrolled in Introductory Psychology at Ryerson University in Toronto, Ontario $\left(M_{\mathrm{age}}=19.89, S D_{\mathrm{age}}=2.88,53\right.$ Females, 19 Males, 1 Non-binary gender $)$ completed the first experimental session. A total of 13 participants dropped out of the study after completing the first experimental session (attrition rate $=17.8 \%$ ). Additionally, one participant completed both days of testing, but an administration error occurred on day 1 which rendered the RK data for the participant unusable on test day 1. Sixty-one participants successfully completed both days of testing $\left(M_{\mathrm{age}}=19.79, S D_{\mathrm{age}}=2.87,42\right.$ Females, 18 Males, 1 Non-binary gender $)$. The justification for a recruitment target of 60 participants was based on previous studies of emotion regulation, in which approximately 40 to 60 participants were recruited for studies in which a similar experimental design was employed (e.g., Dillon et al., 2007; New et al., 2009;

Schmeichel, Volokhov, \& Demaree, 2008). Furthermore, based on an expected medium effect 
size (Cohen's $d=.50$ ) consistent with findings in the emotion regulation and memory literature (Dillon et al., 2007; Richards \& Gross, 1999), power analyses run using G*Power Version 3.0 (Faul, Erdfelder, Lang, \& Buchner, 2007) indicated a required sample size of 54 participants to obtain $95 \%$ power for key pairwise contrasts between the repeated-measures conditions with an alpha $=.05$ (2-tailed).

All participants were recruited through the Ryerson University Psychology Research Pool using the online Sona recruitment system (Sona Systems, Ltd.). Within Sona, a prescreening questionnaire assessed for exclusion criteria including self-reported history of neurological or psychiatric disorders, brain injury, substance abuse, or psychotropic medication use. Only eligible students were able to view the advertisement for this study. Further, on the first test day, all participants completed a demographic and screening questionnaire to capture demographic information to characterize the sample (e.g., age and sex), and to ensure that participants did not endorse any exclusion criteria (see Appendix A). Following data collection, screening questionnaires were reviewed such that only participants with self-reported normal or correctedto-normal vision and hearing, and who are free from current or past neurological and/or psychiatric impairment, were included in the data analysis.

The experiment occurred over two separate testing sessions that were one week apart. One participant was tested per testing session. The study was conducted over a 2-hour period: the first testing session took approximately 1.5 hours to complete and the second testing session took approximately 30 minutes to complete. The incentive for participation in this experiment was two equivalents of course credit, or, two percent of a participant's grade in Introductory Psychology. 
During the first test session, the experimenter began the informed consent process by explaining the study to the participant and providing an informed consent form for the participant to read and sign. Only participants who signed the informed consent form began the experiment. The informed consent form explained the experimental tasks and study design, advised the participants that they could withdraw from the experiment at any time during the testing session (including the option to withdraw their study data from analysis), and informed them that they would receive course credit in Introductory Psychology for participating, even if they choose to leave the experiment early or withdraw their data.

Following the informed consent process, participants completed a modified version of the EMS emotion regulation task (Jackson et al., 2000; see elaboration on the task below) as the encoding phase of the experiment. Next, participants were subject to a 15-minute delay period during which they completed the DASS-21 and a brief working memory task. A 15-minute delay period has been employed in studies of a similar design using the RK procedure (i.e., Patel et al., 2012) and serves to reduce task-specific fatigue that one may experience from viewing emotional images. Moreover, due to the sustained attention required by these "filler" tasks, participants would be unlikely to rehearse the images from the encoding phase (Patel et al., 2012). Following the delay period, participants completed a surprise recognition memory test for half of the study items using a variation of the RK procedure (Migo et al., 2012; Tulving, 1985).

For those participants interested in completing the study, a seven-day delay period followed the completion of the first day of the experiment. The participants then returned to the laboratory after exactly seven days to complete the second part of the experiment, which occurred over a 30-minute period. On this second test day, participants completed the RK procedure using the remaining half of the stimuli encoded on the first day of the experiment that 
was not previously tested on Day 1, as well as the DASS-21, ERQ, TMMS, and the MAAS.

Finally, participants completed two additional brief working memory capacity tasks to characterize the working memory ability of the sample. Following this, participants were fully debriefed on the study objectives, given a debriefing form and the opportunity to ask questions, and thanked for their participation.

Approval for this research study by the Research Ethics Board at Ryerson University was obtained prior to data collection.

\section{Stimuli}

Stimuli were selected from the recently-developed 900-item Open Affective Standardized Image Set (OASIS; Kurdi, Lozano, \& Banaji, 2017). The creation of this standardized image set involved a diverse adult sample of 225 participants rating images on their valence and arousal levels. In the construction sample, both arousal and valence were rated by participants on a scale from $1-7(1=$ very low, $7=$ very high $)$ and valence was rated on a scale from $1-7(1=$ very negative, $7=$ very positive). The benefits of using the OASIS over other established image sets included standardized image sizes; standardized image categories with many items in each category (e.g., human scenes, animals); modern, high-quality colorized images; no associated cost to using the images; a construction sample that comprised diverse adults from the United States; and detailed descriptive information about each image (i.e., valence and arousal ratings for males and females, respectively).

A total of two sets of 120 images each were selected from the OASIS for the purposes of the study. Sixty of the images in each set were selected for the purposes of eliciting negative emotions, such as fear, sadness, or anger, at varying levels of arousal. Descriptive statistics for 
the 120 negative images: $M_{\text {arousal }}=4.21, S D_{\text {arousal }}=0.64$, Range $_{\text {arousal }}: 1.87-5.47 ; M_{\text {valence }} 1.08$, $S D_{\text {valence }}=0.23$, Range $_{\text {valence }}=.42-1.65$.

The remaining 60 images in each set were selected for the purposes of not eliciting emotional responses (i.e., neutral images). Descriptive statistics for the 120 neutral images: $M_{\text {arousal }}=2.84, S D_{\text {arousal }}=0.91$, Range $_{\text {arousal }}: 1.69-4.72 ; M_{\text {valence }} 4.03, S D_{\text {valence }}=0.14$, Range $_{\text {valence }}=3.74-4.26$ (see Figure 1 for an example of a negative image and a neutral image from the OASIS).

The content of the images was equated across neutral and unpleasant stimuli (e.g., human scenes, inanimate objects, nature scenes, and images of animals). In addition, both neutral and unpleasant images were each divided into three subsets for presentation with the three EMS instructions. Further, within and across the two 120-image sets, the subsets of negative stimuli were equated with regards to their content types, the mean valence ratings of the images, and the mean arousal level of the images. Likewise, subsets of neutral stimuli were matched for content and mean valence and arousal ratings $(p s>.05)$.

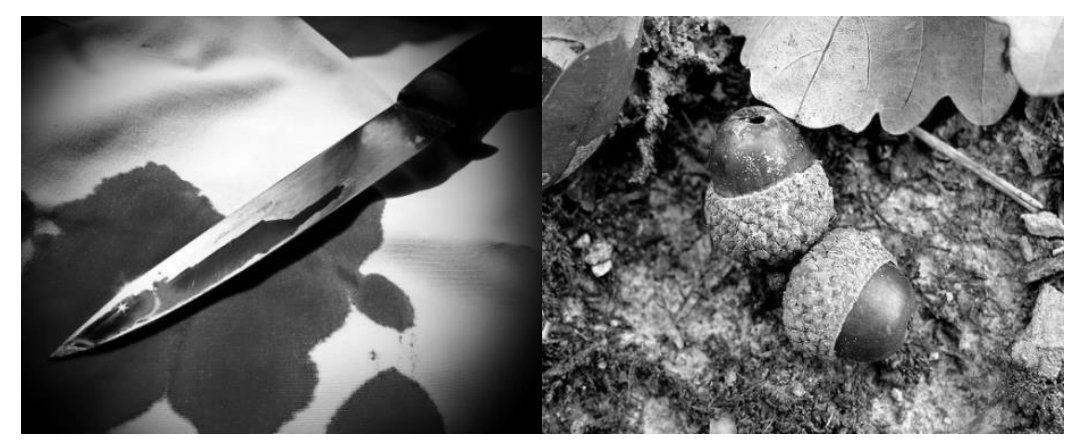

Figure 1. Exemplar unpleasant and neutral photos from the OASIS (Kurdi et al., 2017).

To minimize potential confounds related to particular combinations of stimuli, half of the participants in the study viewed one set of 120 OASIS images during the encoding phase on the first day of testing ("List 1"), while the remaining half of the participants viewed the second set 
of 120 OASIS images ("List 2"). During the recognition memory task on the first day of testing, participants who viewed images from List 1 made "Remember", "Know" or "New" judgments (i.e., RK judgments, as explained below) on a total of 120 images: 60 images that were previously encoded from List 1, and 60 new foil images from List 2 that were not previously seen. On the second day of testing, these same participants made RK judgments on a new set of 120 images: the remaining 60 images previously encoded from List 1, and the 60 remaining foil images from List 2. Similarly, participants who viewed images from List 2 on the first day of testing made RK judgments on a total of 120 images: 60 images that were previously encoded from List 2, and 60 new foil images from List 1 that were not previously encoded. On the second day of testing, these same participants made RK judgments on a new set of 120 images: the remaining 60 images previously encoded from List 2, and the 60 remaining foil images from List 1.

\section{Encoding Task}

A 40-minute version of the computerized EMS task initially developed by Jackson and colleagues (2000) was used in this study during the stimulus encoding phase. During this task, individuals were exposed to either unpleasant or neutral images and were instructed to enhance, maintain, or suppress their emotional responses to the images. Many studies on emotion regulation and emotional memory use primarily unpleasant and neutral images during the encoding task (e.g., Dillon et al., 2007; Jackson et al., 2000; New et al., 2009). As such, the omission of positive images is congruent with much literature in these areas, and is clinically relevant, as the ability to regulate negative emotions is a common treatment goal of many types of psychotherapies (e.g., Linehan, 1993). The images were displayed on a computer running EPrime 2.0 software (Psychology Software Tools [PST], Pittsburgh, PA), a widely-used software 
for designing and conducting psychological experiments involving audio and/or visual stimuli. This task employed a 2 (Stimulus type: unpleasant, neutral) x 3 (EMS instruction condition: enhance, maintain, suppress) within-subjects design.

The task began after three short practice trials to ensure that the participant understood the instructions. See Appendix $B$ for a transcript of the verbal instructions (including for the practice trial) that were administered to participants. Upon confirmation that the instructions were understood, the participant began the experiment. On their computer screen, an image was displayed for a total of 8 seconds (see Figure 2). Four seconds post-stimulus onset, a digitized human voice provided them with an emotion regulation instruction (in parentheses); for all images, participants were asked to either suppress ("suppress"), enhance ("enhance"), or maintain ("look") their emotional response to the image. A fixation cross appeared on the screen for an additional 8 seconds following the disappearance of the image, during which time participants were instructed to continue modulating their emotional response to the initial image. Finally, the fixation cross was replaced with the word "relax" for a total of 4 seconds, indicating that the individual was to stop enhancing, maintaining, or suppressing their emotional response. After the 4 seconds, a new image was shown, and again the participant was instructed to regulate their emotion in response to the image (Jackson et al., 2000).

Three blocks of 40 images were shown to each participant for a total of 120 images (60 unpleasant images and 60 neutral). The images and the emotion regulation cues were pseudorandomized such that neither image valence (e.g., unpleasant or neutral), image category (e.g., person, scene, etc.) nor emotional response instruction (e.g., enhance, maintain, or suppress) was repeated consecutively more than three times (in congruence with Jackson and colleagues, 2000). Thus, each block contained intermixed image and instruction types, such that 
all unpleasant images and neutral images across all image categories were paired with all three emotion regulation cues, and that all combinations of image type, category, and instruction type were shown within each block. Each block of 40 images lasted approximately 14 minutes in duration. After each block, participants were guided to complete a short emotion regulation strategy questionnaire that takes approximately five minutes to complete (as described in the Strategy Questionnaire section below; see Appendix C). As such, the encoding phase of the experiment lasted approximately 60 minutes.

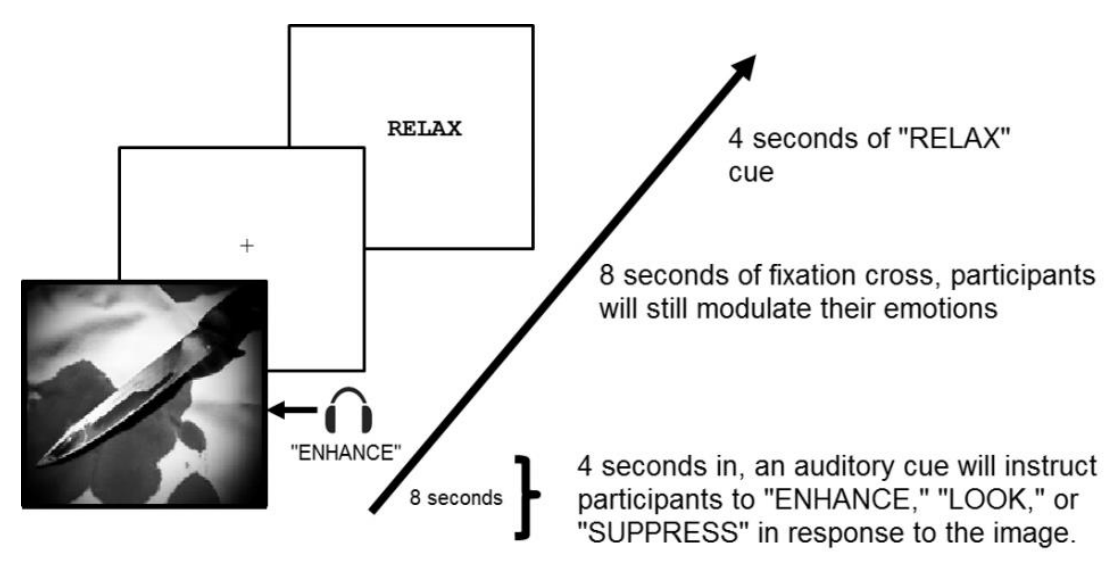

Figure 2. The emotion regulation paradigm adapted from Jackson and colleagues (2000).

During the task, participants viewed an image (either negative or neutral) for a total of eight seconds. Halfway through viewing the image (i.e., after four seconds had elapsed), participants received an auditory instruction to either enhance, maintain (i.e., "look" instruction), or suppress their emotional response to the image. After the image disappeared from the screen, participants were instructed to continue modulating their emotions while a fixation cross was shown for eight seconds. Finally, participants were shown a "relax" screen for four seconds, indicating that they should stop modulating their emotion to the image. 


\section{Strategy Questionnaire}

After viewing each block of 40 images, participants completed a brief computerized questionnaire that assessed what strategies they had used during the preceding block to enhance, maintain, or suppress their emotions, and how successful they were at using those strategies. As such, participants did not evaluate their use of each strategy immediately following their strategy use, but rather after viewing 40 images. This strategy questionnaire allowed participants to select one or more strategies from a list of several common emotion regulation strategies reported in the literature (e.g., Dillon et al., 2007; Jackson et al., 2000; New et al., 2009). Specifically, participants could select one or more of three cognitive reappraisal strategies: Increasing or decreasing the severity of the situation seen in the image, increasing or decreasing the severity of the future outcome of the situation seen in the image, and increasing or decreasing the personal relevance of the image to one's own life. They could also select from one or more of two autonomic strategies: Changing one's facial expression and changing one's breathing/muscle tension. Participants were also provided the option to describe an idiosyncratic strategy that they used.

In addition to selecting emotion regulation strategies used during the preceding 40 images of the EMS task, participants were asked to (1) explain how they used that strategy by entering their response into a textbox and to (2) rate what percentage of the time throughout the block that unique strategy was used. Participants were also given the option to indicate if they did not use an emotion regulation strategy, if they were not aware of what strategy that they used, or if they were not comfortable sharing which strategy they had used. Moreover, participants were asked to rate both how successful they felt while modulating their emotional responses, as well as how much attention they paid to each image when it was paired with each 
respective instruction. Finally, during the second and third blocks of the EMS task, participants were asked whether their emotion regulation strategy use had changed from the previous block and, if so, were invited to type into a textbox to explain why their strategy use changed. See Appendix $C$ for a copy of the strategy questionnaire.

\section{Distraction Period Tasks}

Similar to other studies of emotion regulation(e.g., Dillon et al., 2007, Rasch et al., 2009; Ritchey et al., 2008), participants underwent a 15-minute distraction period following the completion of the encoding task. During this time, participants completed the 21 -item DASS-21 (Lovibond \& Lovibond, 1995; described in the Questionnaires section), as well as a working memory capacity (WMC) task. The purpose of these filler measures was to minimize the possibility that participants were mentally revisiting the images they had viewed during the encoding task; to better characterize participants' levels of depression, anxiety, and stress that may have impacted their ability to regulate their emotions during the task; and to characterize participants' WMC.

Working memory tasks. In a series of studies conducted by Schmeichel, Volokhov, and Demaree (2008) on WMC and the self-regulation of emotional expression and experience, the authors found that individuals with higher levels of WMC were better at both suppressing emotional facial expressions and appraising emotional stimuli in a non-emotional manner as compared to those with lower levels of WMC. Thus, to better characterize the cognitive abilities of our sample, shortened versions of three automated complex span tasks developed by Oswald and colleagues (2015) to assess WMC were completed by participants across two test days. These include the RSPAN, SSPAN, and the OSPAN. The rationale for including these tasks 
include their strong psychometric properties, their balance between domain specific and general WMC, and their brief total administration time (9.7 minutes on average; Oswald et al., 2015).

During the distraction period on the first test day, the RSPAN was administered. During this task, participants read sets of both sensible and non-sensible sentences of varying length (approximately 10-15 words per sentence). Upon reading each sentence, participants were asked to judge whether each sentence makes sense (approximately half of the sentences are sensible), after which they are presented with a single letter. After being presented with several sentences which comprise a "set" (with each set comprised of $4-6$ sentences), participants are asked to recall the letters that had followed the sentences that they had read during that set (Oswald et al., 2015). The average partial-load score for participants who successfully completed the RSPAN with an error rate $\leq 15 \%$ and no technical errors $(n=62)$ was $23.42(S D=4.62)$.

During the second test day, participants completed the SSPAN and the OSPAN. During the SSPAN, participants viewed a total of 42 different matrices comprised of black and white squares that were either symmetrical across the vertical axis or were not. Participants were asked to evaluate if the matrix is vertically symmetrical. After making their evaluation, participants viewed a smaller matrix with several red squares positioned inside. Participants were asked to recall the position of the red squares at the end of each set of matrices that they viewed, with set sizes ranging from 2 matrices to 5 matrices (i.e., 2 to 5 different evaluations). The average partial-load score for participants who successfully completed the SSPAN with an error rate $\leq$ $15 \%$ and no technical errors $(\mathrm{n}=52)$ was $17.12(S D=4.41)$.

During the OSPAN, participants were asked to evaluate whether each of 75 arithmetic operations (e.g., $2+2=4$ ) is true or false. After each evaluation, participants were shown a letter (e.g., "A") that they were asked to recall at the end of a set of arithmetic operations. Set sizes 
varied from $4-6$ equations in each set (i.e., $4-6$ different evaluations). The average partial-load score for participants who successfully completed the OSPAN with an error rate $\leq 15 \%$ and no technical errors $(\mathrm{n}=50)$ was $24.5(S D=5.05)$.

\section{Recognition Task}

After completing the DASS-21 and RSPAN, the experimenter alerted participants to the next task, which involved recalling the images seen during the EMS task. Participants first read computerized instructions on how to make RK judgments (see Appendix D). Next, the experimenter read verbal instructions to the task and gave the participants the opportunity to ask questions. In addition, to determine that these instructions were understood by participants, the participant completed three practice trials with two previously studied images and one new image. During these trials, participants were asked to say aloud their choice of a "Remember," "Know," or "New" response to the experimenter, and to explain how they had made that specific RK judgment. Specifically, participants were evaluated on their understanding that a remember response indicated that they could recollect specific details regarding their previous viewing of the study image (i.e., what they had thought when they first saw it, what order it came in the sequence of images, etc.); that a know response indicated that they were confident that they have seen the image, but could not recollect any specific details about the encoding experience; and that a new response indicated that they had not seen the image before. Once the experimenter was assured that the participant understood the task instructions, the RK procedure began.

During the recognition phase of the experiment, participants were situated at a computer running the RK test using E-Prime 2.0 (PST). During this test, participants viewed a total of 120 images for which they were asked to make RK judgements at a pace of their choosing. Sixty of these images were previously encoded during the EMS task, while the remaining 60 were 
unstudied foil images. Because this task was self-paced, we estimated that the average person would take approximately 4-6 minutes to make RK judgments (e.g., we estimated that it would take 2-3 seconds to make a RK judgment for each of the 120 images.)

The stimuli were presented in a pseudorandom order, such that no image "type" (i.e., specific category of image, valence of image, or old or new image) was repeated more than two consecutive times. Each trial consisted of a slide showing either a foil image or a previously studied image, with text underneath that asked participants to indicate whether the photo was "remembered", "known", or "new" by pressing the appropriate key $(\mathrm{R}, \mathrm{K}$, or N) on the keyboard. Once a response was registered by the computer program, the next slide with a new image would appear, until a total of 120 images are shown.

Participants completed the RK procedure on both test days. On the first test day, 60 images from the photoset viewed by the participant during the encoding phase and 60 foil images were shown. On the second test day, the remaining 60 images from the original photoset as well as a new set of 60 foil images were shown. Thus, each study image was only encoded and subsequently tested once, and foil images were only ever viewed once during the RK procedure. Further, the stimulus sets administered during encoding, as well as the foil images, were counterbalanced across participants.

\section{Questionnaires}

Four questionnaires, in addition to the emotion regulation strategy questionnaire administered during the encoding task, were administered to participants to both better characterize the sample and to examine potential correlates of performance on the RK procedure.

During both testing sessions, participants completed the DASS-21 (Lovibond \& Lovibond, 1995). By administering the DASS-21 twice, the relationship between participants' 
levels of depression, anxiety, and stress over the past week and performance on the RK procedure can be examined per test day. They also completed the ERQ (Gross \& John, 2003), the MAAS (Brown \& Ryan, 2003), and the TMMS (Salovey et al., 1995) questionnaires. All questionnaires were presented in randomized order. These questionnaires were administered on a computer running the survey software, Qualtrics (Qualtrics, Provo, UT). By using computerized versions of these questionnaires, errors made when entering data from a paper form to a computerized version were reduced, as Qualtrics allows the survey data to be exported directly from the website as a data file that can be analysed using statistical software. Altogether, these questionnaires took approximately 20 minutes for participants to complete. Moreover, all questionnaire data were analysed according to standard scoring procedure.

Depression and Anxiety Stress Scales - 21. The DASS-21 (Lovibond \& Lovibond, 1995) is a widely-used 21-item measure of depression, anxiety, and stress that one has experienced over the past week. The instrument comprises three subscales: the depression subscale, which is a measure of low mood and self-esteem; the anxiety subscale, which is a measure of anxious arousal and the physiological manifestations of anxiety; and the stress subscale, which is a measure of agitation and tension. The DASS-21 was administered at the end of the first test day, as well as during the questionnaire administration portion during the second test day.

Day 1. Reliability analyses of the 21-item DASS-21 during the first experimental test session revealed excellent levels of internal consistency as measured by a Cronbach's alpha of .868 on the sample of 71 participants who completed the first test session. Similarly, reliability analyses of each DASS-21 subscale across the first experimental test session revealed excellent 
levels of internal consistency for the depression, anxiety, and stress subscales (7 items each), as measured by Cronbach's alpha $(\alpha=.78, \alpha=.66$, and $\alpha=.75$, respectively).

Day 2. In congruence with reliability results from day 1, overall, the day 2 DASS-21 reliability analyses demonstrated excellent levels of internal consistency as measured by an overall Cronbach's alpha value of .899 on the sample of 58 participants who completed both test sessions. Furthermore, reliability analyses of the DASS-21 subscales across day 2 revealed good levels of reliability for all three subscales, as measured by Cronbach's alpha (depression $\alpha=.85$, anxiety $\alpha=.69$, and stress $\alpha=.78)$.

Emotion Regulation Questionnaire. The ERQ (Gross \& John, 2003) assesses how often participants use cognitive reappraisal and autonomic suppression in their daily life. This 10 -item measure has been validated in undergraduate samples, takes less than 10 minutes to administer, and has strong psychometric properties (Gross \& John, 2003), rendering it a valuable instrument for the purposes described herein. Reliability analyses on the sample of 58 participants who completed both test sessions revealed good levels of internal consistency across both the reappraisal (6 items) and suppression (4 items) subscales of the emotion regulation questionnaire (Gross \& John, 2003), as measured by Cronbach's alpha values of .74 and .71, respectively).

Mindful Attention and Awareness Scale. Mindfulness has been described as a twocomponent process that includes self-regulation of attention and as well as an orientation to experience, such that one adopts a mental stance of curiosity and acceptance to one's own thought processes while being mindful (Bishop et al., 2004). Levels of state and trait mindfulness have been associated with greater capacity to regulate emotions (e.g., Arch \& Craske, 2006) and have thus been integrated into therapies for individuals with difficulties regulating their own emotions (e.g., dialectical behavior therapy for borderline personality disorder; Linehan, 1993). 
The MAAS (Brown \& Ryan, 2003) is a 15-item measure of trait mindfulness. Like the ERQ, the MAAS takes less than 10 minutes to complete, has strong psychometric properties, and has been validated for use in undergraduate samples (Brown \& Ryan, 2003). A reliability analysis of the 58 responses on the 15-item MAAS (Brown \& Ryan, 2003) revealed excellent internal consistency as measured by Cronbach's alpha $(\alpha=.86)$.

Trait Meta-Mood Scale. The 30-item version of the TMMS (Salovey et al., 1995) is a measure of one's capacity to both manage and monitor one's own emotions (Palmer, Gignac, Bates, \& Stough, 2003). The measure comprises three subdomains: Attention to Feelings (i.e., a measure of one's tendency to pay attention to their own emotions), Clarity of Feelings (i.e., a measure of one's ability to understand and distinguish between emotions), and Mood Repair (i.e., a measure of emotion regulation). Like the preceding measures, several studies have demonstrated its brief response time and its strong psychometric properties (e.g., Palmer et al., 2003; Salovey et al., 1995), and have validated its use in undergraduate populations. A reliability analysis of the 58 responses on the TMMS revealed an overall excellent internal consistency as measured by Cronbach's alpha $(\alpha=.86)$. Similarly, good levels of internal consistency were also revealed across all the clarity (11 items), repair (6 items), and attention (13 items) subscales ( $\alpha=$ $.87, \alpha=.78$, and $\alpha=.77$, respectively).

\section{Data Analysis}

Emotion regulation strategy questionnaire. Emotion regulation strategy questionnaire scores were computed via three methods. First, the raw percentage values for each of the five strategies (reappraisal strategies: reappraisal of the image situation, reappraisal of the image future outcome, reappraisal of the image's personal relevance to oneself; autonomic strategies: facial expression changes and breathing changes) during the first block of the emotion regulation 
strategy questionnaire, per instruction to either enhance or suppress emotional responses, were used as a measure of "spontaneous" or "trait-like" use of emotion regulation strategies, as participants were not previously introduced to these strategies prior to viewing the questionnaire. Second, the raw percentage values for each of these strategies was summed across all three blocks, per instruction to enhance or suppress, as a measure of overall strategy use, which may reflect "state-like" emotion regulation strategy use, as participants were introduced to additional strategies that they could choose to employ throughout the remainder of the experiment. Third, these total raw percentage values of each of the three reappraisal strategies per instruction to enhance or suppress were summed into a "total reappraisal" score, and the two autonomic strategies per instruction to enhance or suppress were summed into a "total autonomic" score. As such, these three different measures of emotion regulation strategy use (i.e., spontaneous strategy use during the first block of the questionnaire, overall strategy use throughout the experiment, and total reappraisal and total autonomic strategy use overall) were correlated with various aspects of recognition memory and measures of daily life emotion regulation use, as described in the Results section below.

Remember/Know Procedure. In the current study, correct remember and know responses are used as proxies for recollection and familiarity, respectively. These responses were used as proxies because it is not possible to calculate standard recollection and familiarity estimates (per Yonelinas, 1998) given the current study design. The recollection and familiarity estimate formulae involve the calculation of false alarms for each condition, which is not possible in this study, because it used a within-subjects design with the encoding instructions serving as a key factor of interest when determining memory outcomes. For example, it was not possible for participants in this study to incorrectly indicate that they remembered an image that 
was new for the enhance instruction, because there is no way for a new image to be paired with an emotion regulation instruction. As such, recollection and familiarity estimates were not calculated.

Recognition memory performance indices included hit rates, which represent the proportion of remember or know responses assigned to a previously-studied image, and false alarm rates (FAR), which represent the proportion of remember or know responses assigned to a new image. These raw hit rates were adjusted based on a recommendation by Snodgrass and Corwin (1988), such that 0.5 was added to each numerator and 1 to each denominator. This adjustment was added to ensure that when calculating response bias $\left(\mathrm{B}_{\mathrm{r}}\right)$, which represents the FAR / [1 - (hit rate-FAR)], a nonzero value would be in the denominator. $B_{r}$, which reflects "the decision rule an individual uses when faced with uncertainty on recognition memory tasks," (Huh, Kramer, Gazzaley, \& Delis, 2006, p.1) ranges from $0-1$, with values less than 0.5 representing liberally responding (i.e., the tendency to report new images as old) and values greater than 0.5 representing conservatively responding (i.e., the tendency to report old images as new). A measure of recognition accuracy, the "discrimination index" $\left(\mathrm{P}_{\mathrm{r}}\right)$, was also calculated based on a suggestion by Snodgrass and Corwin (1988) as well as by Yonelinas and colleagues ("recollection formula B"; 1998). This value represents the hit rate - FAR for remember and know responses, respectively, and, as such reflects one's ability to discriminate between old and new images on a scale from -1 to +1 , with higher values reflecting an increased ability. As such, a simpler formula for $\mathrm{B}_{\mathrm{r}}=\mathrm{FAR} /\left(1-\mathrm{P}_{\mathrm{r}}\right)$. For the reasons outlined above, $\mathrm{P}_{\mathrm{r}}$ and $\mathrm{B}_{\mathrm{r}}$ were calculated overall, rather than per emotion regulation task instructions provided.

Several sets of correlational analyses were run to determine the influence of emotion regulation strategy use on remember and know hit rates. The percentage of time each enhance 
emotion regulation strategy was used during the first set of images was correlated with memory for negative images paired with the enhance instruction on day 1 and day 2. Correlational analyses were also run between the total percentage of time each enhance emotion regulation strategy was used across all negative images and memory for negative images paired with the enhance instruction on day 1 and day 2. Finally, parallel sets of correlational analyses were run between the suppress emotion regulation strategies (both spontaneous and overall) and memory for the negative images paired with the suppress instruction. Correlational analyses involving emotion regulation strategy use on memory were conducted on negative images only, because the questionnaire had only asked participants to identify the strategies that they had used to regulate their emotions while viewing negative images. Moreover, correlational analyses were conducted between images paired with a specific instruction (i.e., enhance) and emotion regulation strategies for that same instruction to both reduce the family-wise error rate that arises with multiple comparisons, and because the ER questionnaire instructions asked participants to identify the strategies they used for images paired with each respective instruction only. Only correlations with $r$-values greater than 0.3 (in the top third of effect sizes reviewed by Hemphill, 2003) and with a $p$-value less than .05 are reported in the Results section below to decrease the likelihood of Type I Error. All correlation tables are reported in Appendix F.

Working memory capacity. As per recommendations by Oswald and colleagues (2015), partial-credit task scores were computed and analyzed separately for all three WMC measures. Subsequently, a WMC composite score was created using data from participants who completed all three tasks without any technical errors, and who demonstrated an error rate of equal to or less than $15 \%(\mathrm{n}=41)$. The WMC composite score comprises the average of the standardized (i.e., $\mathrm{z}$ - 
score) partial-credit task scores. Given that the WMC composite score is the average of three zscores, its mean and SD are expected to equal 0.0 and 1.0, respectively.

Data cleaning and screening. All data were subject to a cleaning and screening procedure. Data were first visually inspected for any obvious errors and then were converted to standardized z-score values (relative to the sample mean and standard deviation values). Any zscores that were greater than 3.29 or less than -3.29 were considered outliers, as per recommendations by Tabachnick and Fidell (2006). Data from three individuals were removed for the purposes of analysis due to significant outliers based on these cut-offs across two or more memory measures. Further, it must be noted that because 13 participants did not complete the second day of testing after the one-week delay, the sample size used throughout the results section changes depending on the level of analysis. Next, the distributional properties of the main variables of interest (i.e., memory measures and questionnaire scores) were assessed to determine whether they violate the assumptions of normality; the skewness and kurtosis values of these variables were examined. As per recommendations by Tabachnick and Fidell (2006), the critical ratio (i.e., test statistic/standard error) was computed, with the same $z= \pm 3.29(p<.001)$ cut-off used. An alpha value of .05 was used when evaluating statistical significance of the main analyses of experimental interest.

As expected, most of the memory outcome measures did not meet the assumptions of normality. For variables that were non-normally distributed and involved in pairwise contrasts, these contrasts were run using non-parametric tests (data not shown) to corroborate the parametric test findings reported below in the Results section. 


\section{Remember/Know Procedure}

\section{Results}

$P_{r}$ and $B_{r}$. Analyses of $P_{r}$ using the sample of 58 participants who completed both days of testing revealed moderate levels of recognition accuracy for remember responses on the first test day, $M=.59, S D=.18$, with less accurate memory for know responses on the first test day, $M=.13, S D=.21$. On the second test day, recognition accuracy for remember responses significantly decreased, mean difference $=.33, S E=.02, p<.001$, while recognition accuracy for know responses significantly increased, mean difference $=.05, S E=.03, p=.040$.

Analyses of $\mathrm{B}_{\mathrm{r}}$ indicate that participants tended to respond "old" to items when they were unsure over both test days. On the first test day, response bias was approximately equal across both remember $(M=.17, S D=.14)$ and know responses $(M=.17, S D=.09)$. Over the second test day, $\mathrm{B}_{\mathrm{r}}$ was lower for remember responses, $M=.06, \mathrm{SD}=.04$, than know responses, $M=$ $.15, S D=.08$. These results indicate participants were more likely to assign a know response, rather than a remember response, to a new image. Moreover, these participants were more liberal when assigning remember responses on Day 2 than on Day 1.

Remember and know hit rate analyses. The influence of the emotion regulation instructions on hit rates across both test sessions was measured using a 2 (Day: 1,2$) \times 2$ (Valence: Negative, Neutral) $\times 3$ (Instruction: enhance, maintain, suppress $) \times 2$ (Response: Remember, Know) within-subjects ANOVA, on a sample of 58 participants who completed both test days and whose data did not contain significant outliers. The resulting $2 \times 2 \times 3 \times 2$ ANOVA revealed that the main effects of Day, $F(1,57)=450.69, p<.001, \eta_{p}{ }^{2}=.89$, and Response, $F(1$, $57)=32.07, p<.001, \eta_{p}{ }^{2}=.36$, were qualified by a Day $\times$ Response interaction, $F(2,114)=$ $82.12, p<.001, \eta_{p}^{2}=.59$. Simple effects analyses revealed that during the first experimental session, participants' hit rates were significantly higher for remember responses than know 
responses, mean difference $=.34, S E=.02, p<.001$. However, during the second experimental session, there was no significant difference between participants' hit rates for remember and know responses, mean difference $=-.03, S E=.22, p=.193$.

No main effects of Instruction, $F(2,114)=0.65, p=.527, \eta_{p}^{2}=.01$, nor Valence, $F(1,57)$ $=1.29, p=.260, \eta_{p}{ }^{2}=.02$, were revealed. However, a significant interaction between Valence and Response did emerge, $F(1,57)=23.76, p<.001, \eta_{p}{ }^{2}=.29$. Simple effects analyses revealed that for negative images, remember hit rates were significantly higher than know hit rates, mean difference $=.25, S E=.03, p<.001$ This effect also held for neutral images, though the mean difference was less than half the size, mean difference $=.11, S E=.03, p=.010$.

No other significant two-way interactions emerged: Day $\times$ Instruction, $F(2,114)=0.12$, $p=.89, \eta_{p}{ }^{2}<.01 ;$ Valence $\times$ Instruction, $F(2,114)=0.66, p=.518, \eta_{p}{ }^{2}=.01 ;$ Response $\times$ Instruction, $F(2,114)=1.52, p=.223, \eta_{p}{ }^{2}=.03$. Moreover, although the three-way Day $\times$ Instruction $\times$ Response interaction was nonsignificant, $F(2,114)=1.75, p=.117, \eta_{p}^{2}=.03$, a significant three-way Day $\times$ Valence $\times$ Response interaction was revealed, $F(2,114)=5.73, p=$ $.020, \eta_{p}{ }^{2}=.09$. For clarity, this interaction is decomposed by experimental session (day) below. The four-way Day $\times$ Valence $\times$ Instruction $\times$ Response interaction was also nonsignificant, $F(2$, 114) $=0.04, p=.959, \eta_{p}^{2}<.01$.

Day 1 ANOVA. The influence of the emotion regulation task on remember and know hit rates was analyzed via a 3(Instruction: Enhance, Maintain, Suppress) $\times 2$ (Valence: Negative, Neutral $) \times 2$ (Response: Remember, Know) factorial within-subjects ANOVA on the total sample of 71 participants who completed day 1 of testing (without significant outliers) to maximize the use of the available data. Although the main effect of Instruction was not significant in the overall ANOVA, it was included in these decomposed ANOVAs due to its theoretical 
importance for the study, and because the effect of Instruction may have been masked by the differences across the other conditions in the omnibus ANOVA. The resulting $3 \times 2 \times 2$ ANOVA revealed a main effect of Response, $F(1,70)=90.51, p<.001, \eta_{p}{ }^{2}=.56$, which was qualified by a Response $\times$ Valence interaction, $F(1,70)=23.29, p<.001, \eta_{p}{ }^{2}=.25$. Consistent with hypothesis 1, simple effects analyses revealed an EEM effect, such that for images that were assigned a remember response by participants, more of these images were negative than neutral, mean difference $=.09, S E=.02, p<.001$. By contrast, for images that were assigned a know response by participants, more of these were neutral than negative, mean difference $=.08, S E=$ $.02, p<.001$ (see Figure 3).

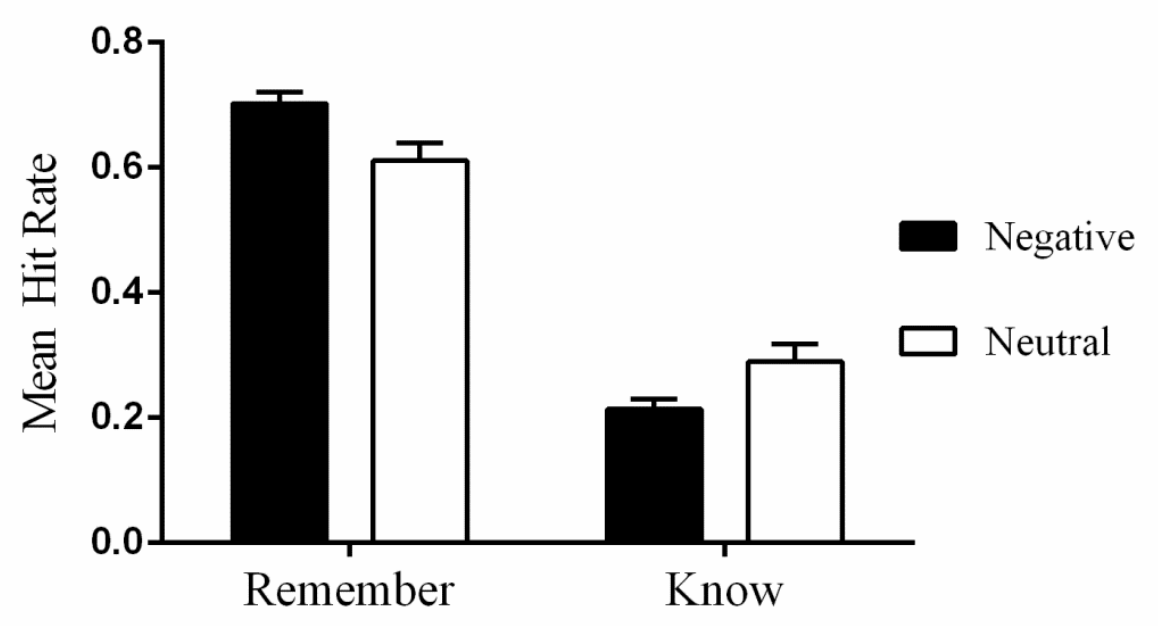

Figure 3. Mean hit rate values for correct remember and know responses, for both negative and neutral images on Day 1 (error bars $=\mathrm{SEM})$.

The $3 \times 2 \times 2$ ANOVA also revealed that the main effect of Response was qualified by an Instruction $\times$ Response interaction, $F(2,140)=4.50, p=.013, \eta_{p}{ }^{2}=.06$. Simple effects analyses revealed that old images, regardless of instruction, were more often assigned a remember response than a know response $(p<.001$ for all comparisons between remembered and known images per instruction). Consistent with hypothesis 3, the images that were paired with the 
enhance instruction were better remembered than images paired with the maintain instruction, mean difference $=.04, S E=.02, p=.015$, and the suppress instruction, mean difference $=.04$, $S E=.01, p=.007$; see Figure 4 . There was no significant difference on remember responses between the suppress and maintain instructions, mean difference $<.01, S E=.02, p=.970$. In an examination of know responses, images paired with the maintain instruction were more often reported as know responses than images paired with the enhance instruction, mean difference $=$ $.04, S E=.01, p=.010$. There was no significant difference between maintain and the suppress instructions, mean difference $=.01, S E=.01, p=.385$, nor between the enhance and suppress instructions, mean difference $=-.02, S E=.01, p=.067$.

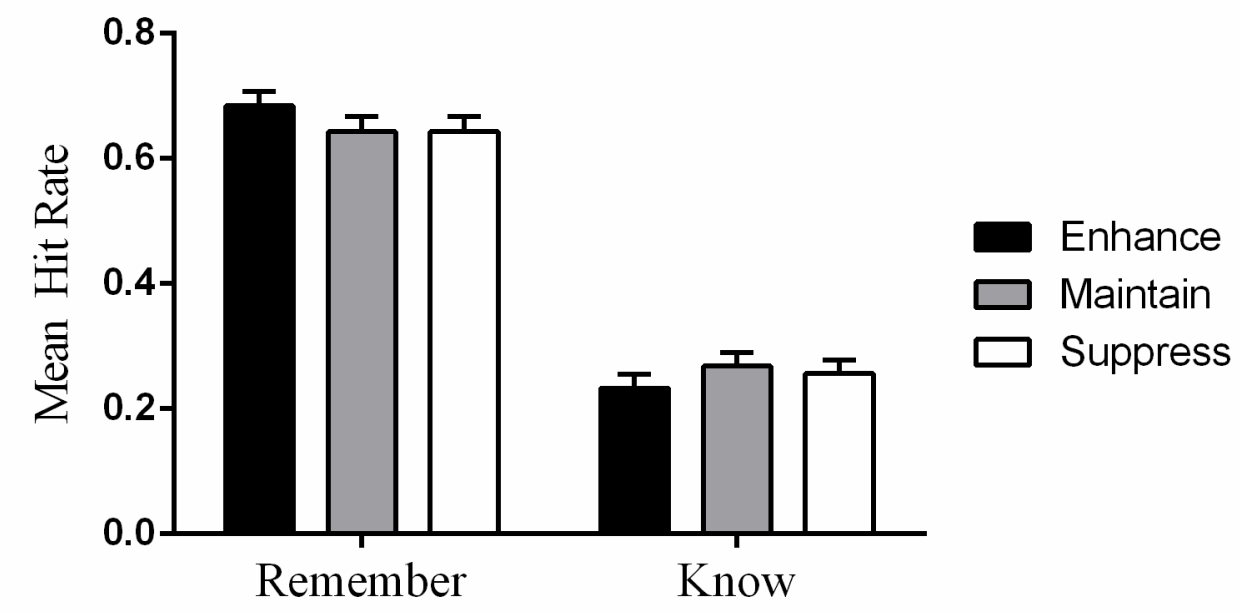

Figure 4. Mean hit rate values for correct remember and know responses, for the enhance, maintain, and suppress instructions during the EMS task on Day 1 (error bars = SEM).

There were neither significant main effects of Valence, $F(1,70)=3.22, p=.077, \eta_{p}{ }^{2}=$ .04 , nor Instruction, $F(2,140)=1.26, p=.288, \eta_{p}{ }^{2}=.02$. There was no significant Valence by Instruction interaction, $F(2,140)=0.63, p=.532, \eta_{p}{ }^{2}<.01$. The three-way Valence $\times$ Instruction $\times$ Response interaction was also nonsignificant, $F(2,140)=0.44, p=.643, \eta_{p}{ }^{2}<.01$. 
Day 2 ANOVA. The influence of the emotion regulation task on hit rates was analyzed via a 3(Instruction: Enhance, Maintain, Suppress $) \times 2($ Valence: Negative, Neutral $) \times$ 2(Response: Remember, Know) factorial within-subjects ANOVA on the sample of 58 participants who completed both test days. The sample size decreased due to attrition after the first experimental session, as described in the Methods section. The resulting $3 \times 2 \times 2$ ANOVA revealed no significant main effects: Valence, $F(1,57)=.12, p=.732, \eta_{p}{ }^{2}<.01$, Instruction, $F(2,114)=.08, p=.922, \eta p^{2}<.01$, Response, $F(1,57)=0.02, p=.896, \eta_{p}^{2}<.01$. However, a significant Response $\times$ Valence interaction was revealed, $F(1,57)=11.04, p=.002, \eta_{p}{ }^{2}=.16$. Simple effects analyses revealed that for images that were assigned a remember response, more of these images were negative than neutral, mean difference $=.05, S E=.02, p=.002$. By contrast, for images that were assigned a know response, more of these were neutral than negative, mean difference $=.05, S E=.02, p=.005$, replicating Day 1 (See Figure 5).

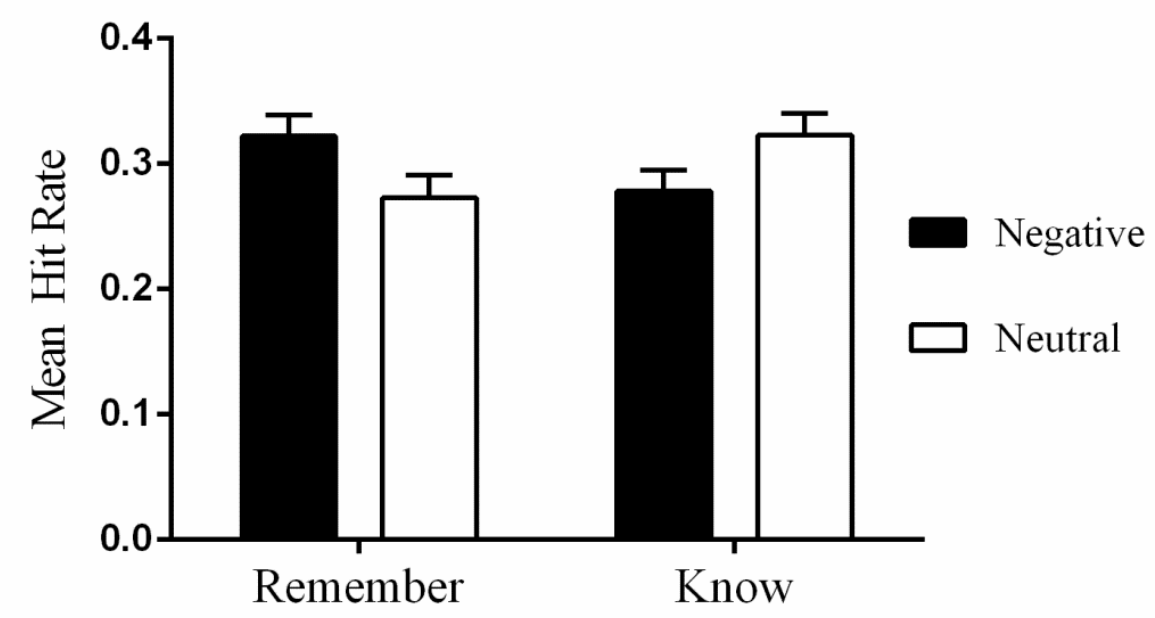

Figure 5. Mean hit rate values for correct remember and know responses, for both negative and neutral images on Day 2 (error bars $=\mathrm{SEM})$. 
No other interaction was significant: Valence $\times$ Instruction, $F(2,114)=.16, p=.852, \eta_{p}{ }^{2}$ $<.01$; Instruction $\times$ Response, $F(2,114)=.08, p=.926, \eta_{p}^{2}<.01$, Valence $\times$ Instruction $\times$ Response, $F(2,114)=.04, p=.960, \eta_{p}{ }^{2}<.01$.

\section{False Alarm Analysis}

Overall, false alarm analyses revealed that participants selected know responses more often than remember responses when incorrectly endorsing new items as old, regardless of test day or image valence. See Appendix $E$ for a more detailed description of these analyses.

\section{Emotion Regulation Strategy Use}

During the emotion regulation task, $63.0 \%$ of the total sample of 73 participants used at least one reappraisal strategy while enhancing their emotions, whereas $35.6 \%$ of the sample used at least one autonomic strategy while enhancing their emotions. By contrast, $54.8 \%$ of the sample used at least one reappraisal strategy while suppressing their emotions, whereas $38.4 \%$ of the sample used at least one autonomic strategy while suppressing their emotions.

Paired sample t-tests on spontaneous strategies used by participants while viewing the first set of images (Block 1) revealed significant differences in strategy use between instruction conditions. Specifically, participants reported using the future outcome strategy as well as idiosyncratic approaches more when instructed to suppress their emotions than when instructed to enhance. Conversely, participants reported using significantly more of the personal relevance strategy when instructed to enhance their emotions than suppress.

Paired sample t-tests on strategies used by participants overall (total across blocks) also revealed a significant difference between the percentage use of the personal relevance strategy, such that more individuals used this strategy when asked to enhance than when instructed to 
suppress. Analysis of the overall strategy use also revealed that more participants used idiosyncratic approaches when suppressing their emotions than when enhancing (See Table 1).

Table 1

Emotion Regulation Strategy Use During Block 1 and Totalled Across Blocks of the EMS Task

\begin{tabular}{|c|c|c|c|c|c|c|c|c|c|c|c|c|}
\hline \multirow[t]{2}{*}{ Strategy } & \multicolumn{2}{|c|}{$\begin{array}{l}\text { Enhance } \\
\text { Block } 1\end{array}$} & \multicolumn{2}{|c|}{$\begin{array}{l}\text { Suppress } \\
\text { Block } 1\end{array}$} & \multirow[b]{2}{*}{$t$} & \multirow[b]{2}{*}{$P$} & \multicolumn{2}{|c|}{$\begin{array}{c}\text { Enhance } \\
\text { Total }\end{array}$} & \multicolumn{2}{|c|}{$\begin{array}{c}\text { Suppress } \\
\text { Total }\end{array}$} & \multirow[b]{2}{*}{$t$} & \multirow[b]{2}{*}{$p$} \\
\hline & $M$ & $S D$ & $M$ & $S D$ & & & $M$ & $S D$ & $M$ & $S D$ & & \\
\hline $\begin{array}{l}\text { Severity } \\
\text { Future }\end{array}$ & 24.77 & 34.68 & 20.00 & 33.89 & 0.94 & .348 & 55.56 & 95.00 & 39.45 & 84.08 & 1.35 & .180 \\
\hline $\begin{array}{l}\text { Outcome } \\
\text { Personal }\end{array}$ & 10.60 & 24.26 & 18.33 & 32.51 & -2.05 & .045 & 27.21 & 67.13 & 33.44 & 76.41 & -.716 & .477 \\
\hline $\begin{array}{l}\text { Relevance } \\
\text { Facial }\end{array}$ & 34.19 & 35.81 & 11.64 & 25.24 & 4.69 & $<.001$ & 72.05 & 94.21 & 27.19 & 65.01 & 3.52 & .001 \\
\hline Expression & 14.59 & 25.90 & 17.48 & 31.91 & 0.656 & .514 & 40.77 & 83.31 & 35.84 & 81.12 & .62 & .540 \\
\hline Breathing & 22.82 & 31.88 & 22.74 & 37.01 & 1.05 & .299 & 39.85 & 85.12 & 47.55 & 91.57 & -1.23 & .223 \\
\hline Other & 66.67 & 32.60 & 77.08 & 27.17 & -2.81 & .006 & 14.85 & 47.28 & 33.74 & 70.14 & -2.29 & .026 \\
\hline
\end{tabular}

Note: Data represent reported percentages of strategy use; $t$ and $p$ values reflect results from paired $t$ tests $(\mathrm{N}=73)$.

Frequency analyses of the emotion regulation strategies used during the first set of 40 images viewed during the EMS task revealed that $83.6 \%$ of participants used more than one reappraisal strategy when enhancing their emotions during the first block of images. Conversely, 15.1\% used more than one autonomic strategy when enhancing their emotions. By contrast, $16.4 \%$ of participants used more than one reappraisal strategy when suppressing their emotions, and $12.3 \%$ used more than one autonomic strategy when suppressing their emotions during the first block of images. Moreover, during the first block of images that were enhanced, $45.2 \%$ of participants used at least one reappraisal strategy and one autonomic strategy. By contrast, for images paired with the suppress instruction during the first block of images, $27.4 \%$ of participants used at least one reappraisal strategy and one autonomic strategy. See Table 2 for descriptive statistics on the amounts of emotion regulation strategies used during the EMS task. 
Table 2

Number of Emotion Regulation Strategies Used During Block 1 of EMS Task and Overall

\begin{tabular}{ccccc} 
& $M$ & SD & Min & Max \\
\hline Reappraisal Strategies - Enhance Block 1 & 1.22 & 0.77 & 0 & 3 \\
Autonomic Strategies - Enhance Block 1 & 0.73 & 0.71 & 0 & 2 \\
Reappraisal Strategies - Enhance Overall & 3.56 & 2.09 & 0 & 9 \\
Autonomic Strategies - Enhance Overall & 1.90 & 1.88 & 0 & 6 \\
Enhance Strategies Overall & 5.89 & 2.81 & 3 & 15 \\
Reappraisal Strategies - Suppress Block 1 & 0.86 & 0.75 & 0 & 3 \\
Autonomic Strategies - Suppress Block 1 & 0.64 & 0.69 & 0 & 2 \\
Reappraisal Strategies - Suppress Overall & 2.56 & 2.00 & 0 & 9 \\
Autonomic Strategies - Supress Overall & 1.92 & 1.83 & 0 & 6 \\
Suppress Strategies Overall & 5.11 & 2.36 & 1 & 12
\end{tabular}

Additional analyses of the emotion regulation strategy questionnaire revealed that when evaluating the amount of attention they paid during the EMS task on a scale from 1 - 5 (with "1" indicating "None at all" and " 5 " indicating "A great deal"), on average, participants rated the level of attention they paid during the EMS task as $3.45\left(S D_{\text {attention }}=0.58\right.$, Range $_{\text {Attention }}=2.33-$ 5.00). Similarly, when evaluating how successful they were at regulating their emotions during the EMS task on a scale from $1-5$ (with " 1 " indicating "Not successful at all" and " 5 " indicating "Extremely successful", on average, participants rated their success as $3.47\left(S D_{\text {success }}=\right.$ 0.62, Range $\left.e_{\text {success }}=1.83-4.83\right)$.

Further analyses of the emotion regulation strategy questionnaire revealed that after viewing the second block of images, $30.1 \%$ of the sample reported that they had changed the strategies that they had initially used to enhance their emotions. Similarly, after viewing the third block of images, $24.7 \%$ of the sample reported that they had changed the strategies that they had used to enhance their emotions during the second block. With regards to the strategies used while asked to suppress their emotions, after viewing the second block of images, $34.2 \%$ of the sample 
reported that they had changed the strategies that they had initially used to suppress their emotions. Finally, after the third block, $24.5 \%$ of the sample reported that they had changed the strategies that they had used to enhance their emotions during the second block. Emotion regulation strategy change is also evident in Table 2 when comparing block 1 to overall strategy use, per instruction condition.

A correlational analysis between the total reappraisal strategy use (i.e., the summed percentage amount of reappraisal strategies used) for enhance and the total reappraisal strategy use for suppress revealed a significant positive relationship, $r(58)=.52, p<.001$. These results indicate that participants who used reappraisal strategies when asked to enhance also tended to use these strategies when asked to suppress. A parallel analysis between the total autonomic strategy use for enhance and the total autonomic strategy use for suppress also revealed a significant positive relationship, $r(58)=.77, p<.001$, again indicating that participants who used autonomic strategies when asked to enhance also tended to use these strategies when asked to suppress.

In summary, participants reported feeling successful at both paying attention to the EMS task as well as using various strategies to regulate their emotions while viewing negative images. When analysing the results of the emotion regulation strategy survey, it was clear that there were marked differences in strategy use between instructions; over $85 \%$ of the sample reported using more than one cognitive reappraisal strategy while enhancing their emotions, while only $16.4 \%$ of participants reported using more than one reappraisal strategy when instructed to suppress their emotions. However, the type of strategies (i.e., reappraisal or autonomic strategies) used during the enhance instruction was predictive of the type strategies used during the suppress instruction. Additionally, approximately one-third of the sample changed emotion regulation 
strategies during the second block of images, suggesting that the sample was flexible in their strategy use. These results suggest that the enhance instruction may lend itself better to cognitive flexibility than suppression.

\section{Working Memory Capacity}

Correlational analyses between the three WMC z-scores revealed a significant positive relationship between performance on the RSPAN and SSPAN tasks $(r(41)=.34, p=.029$. The relationship between the OSPAN and the RSPAN and SSPAN tasks were not significant ( $p$ s > $.05)$.

Correlations between WMC and RK memory performance. Despite initial predictions, no significant correlations were revealed between the composite WMC score and performance on the RK task $(r \mathrm{~s}<.3, p \mathrm{~s}>.05)$.

Correlations between WMC and emotion regulation strategy use. In addition to WMC and memory performance, correlational analyses between the WMC z-scores, the WMC composite score, and of the use of specific emotion regulation strategies during the EMS task were conducted. A significant negative relationship between performance on the OSPAN and the overall use of the personal relevance strategy to suppress emotions was revealed, $r(41)=-.403, p$ $=.009$. Additionally, a significant negative relationship between the WMC composite score and the use of the severity strategy for suppress during the first block of images was revealed, $r(41)=$ $-.422, p=.006$. The strength of this relationship may be due to the SSPAN z-score score specifically, because a significant negative relationship between the performance on the SSPAN and this strategy was also revealed, $r(41)=-.545, p<.001$. No other correlation was significant $(p \mathrm{~s}>.05)$. 
Furthermore, correlational analyses between WMC z-scores, the WMC composite score, and measures of emotion regulation in daily life (i.e., DASS-21, TMMS, MAAS, and the ERQ) were also conducted. A significant positive relationship between performance on the SSPAN and the suppress subscale of the ERQ was revealed, $r(41)=.36, p=.022$. No other correlation was significant $(p s>.05)$.

\section{Correlations Between Emotion Regulation Strategy Use and Questionnaire Data}

Correlational analyses between both spontaneous (i.e., "trait-like" emotion regulation strategy use, as measured by the strategies used during the first block of images) and total emotion regulation strategy use during the EMS task and measures of emotion regulation in daily life (i.e., DASS-21, TMMS, MAAS, and the ERQ) were also conducted (See Appendix F).

For images paired with the instruction to enhance, a significant positive relationship between the use of the personal relevance strategy for enhance during the first block of images and the DASS-21 anxiety scale was revealed, for the first test day only, $r(58)=.39, p=.003$. Significant positive relationships between the overall breathing strategy as well as overall autonomic strategy use (for images that were paired with the enhance instruction) with the DASS-21 anxiety scale on day 2 also emerged, $r(58)=.41, p=.002$, and $r(58)=.36, p=.005$, respectively. Moreover, the overall autonomic strategy use for enhance was positively correlated with the DASS-21 stress scale, also on day $2, r(58)=.32, p=.013$.

For images paired with the instruction to suppress, a significant negative relationship between the use of the severity strategy for suppress during the first block of images and the ERQ suppress subscale was revealed, $r(58)=-.34, p=.008$. Moreover, a significant negative relationship was revealed between the total percentage use of autonomic emotion regulation strategies for images paired with the instruction to suppress and the repair subscale of the 
TMMS, $r(58)=-.31, p=.018$. A significant positive relationship was revealed between the overall summed percentage of the breathing strategy for images that were paired with the suppress instruction and the DASS-21 anxiety scale on day $1, r(58)=.37, p=.004$. Like the results seen for the enhance instruction, significant positive relationships between the overall breathing strategy as well as overall autonomic strategy use (for images that were paired with the suppress instruction) with the DASS-21 anxiety scale on day 2 emerged, $r(58)=.43, p=.001$, and $r(58)=.38, p=.003$, respectively. Moreover, the overall breathing strategy use and the overall autonomic strategy use for suppress were positively correlated with the DASS-21 stress scale on day $2, r(58)=.35, p=.007$, and $r(58)=.32, p=.014$, respectively. No other correlations were both greater than $r(58)=0.3$ and statistically significant $(p<.05)$.

\section{Correlations between Emotion Regulation Strategy Use and Memory Outcomes}

Despite initial predictions (hypotheses 4 and 5), correlational analyses revealed few significant relationships between emotion regulation strategy use and memory outcomes. Furthermore, because these correlations were not in expected directions and were part of many analyses, it is likely that several of these results are attributable to Type I error and should be interpreted with caution.

Several correlational analyses were conducted on the relationship between emotion regulation strategy use during the EMS task and memory outcomes. One correlational analysis revealed a significant negative relationship between the use of the reappraisal strategy "to increase the severity of the situation" during the viewing of images paired with the enhance instruction and the know hit rate for negative images that were enhanced, on the second day of testing only, $r(58)=-.32, p=.013$. A significant positive relationship was also revealed between the percentage use of the future outcome strategy during the first block of images (that were 
paired with the suppress instruction) and remember responses on Day 2 only, $r(58)=.33, p=$ .012 .

Correlational analyses were also conducted on the relationship between daily-life emotion regulation strategy use and memory outcomes. A significant negative relationship was revealed between the TMMS clarity subscale and Day 2 know responses for images paired with the enhance instruction, $r(58)=-.40, p=.002$. Lastly, a significant negative relationship between DASS-21 anxiety subscale on day 1 and day 1 remember responses for images paired with the suppress instruction was also revealed, $r(58)=-.31, p=.019$. No additional correlations were both greater than $r(58)=.30$ and statistically significant $(p<.05)$. In a follow-up analysis, hit rates were collapsed across remember and know responses to assess whether overall hits might prove more sensitive; however, no emotion regulation strategies correlated significantly with memory with an effect size of greater than $r(58)=.30$.

\section{Discussion}

The main objective of this experiment was to examine how the spontaneous use of emotion regulation strategies influences incidental recognition memory for emotional images. In this pursuit, several interesting patterns emerged. First, as expected, the emotional enhancement of memory effect was replicated. Second, the emotion regulation task instructions appeared to differentially influence participants' reported remember and know responses. Third, there appeared to be no relation between emotion regulation strategies used at encoding and subsequent recognition memory for emotional images. Overall, these findings suggest that instructions to regulate emotional responses to both negative and neutral images differentially affect recognition memory for these images. These findings will be discussed in more detail in 
the following sections, followed by a discussion of the experiment's limitations as well as future directions for research on emotion regulation and memory.

\section{Emotional Enhancement of Memory}

Broadly, the results of this experiment replicated the EEM effect, which posits that emotional events are better remembered than neutral ones (Sommer et al., 2008). Indeed, across both test days, negative images were more frequently correctly assigned a remember response than neutral images, whereas neutral images were more frequently correctly assigned a know response than a remember response. This indicates that participants reported greater recollection for negative images than neutral ones across both test days, in congruence with the EEM effect. Moreover, false alarm analyses revealed opposite effects to those seen in the hit rate analyses, such that know responses were more often selected than remember responses, regardless of test day or image valence. These results suggest that participants were not assigning remember responses to emotional images based on their emotional content, meaning that it is unlikely an emotional response bias explains the EEM effect seen in this study.

Based on consolidation theories of EEM (Sharot et al., 2007; Sharot \& Yonelinas, 2008; Sommer et al., 2008) it was expected that recollection would decay more slowly for emotional than neutral images, yielding a larger EEM after the one-weeklong delay. This prediction was not supported. The one-week delay, however, did differentially affect participants' correct remember and know responses, regardless of image valence. Indeed, while there were more correct remember responses than correct know responses assigned during the first test day, there was no significant difference between these two responses after the delay period. Moreover, although remember responses were halved in magnitude from immediate test to the one-week delay, know responses increased, albeit slightly, after the delay period. The findings are in 
congruence with those from a review by Yonelinas (2002, p. 500, Table 13), which reports the hit rate results of seven experiments that had also employed the RK procedure both immediately and following a one-week delay period; the results of these studies also demonstrate sharper decreases in correct remember hits than know hits from immediate test to the test conducted after the one-week delay. However, even though the raw correct know response hit rate values are similar as those seen at immediate test, the familiarity estimates also tend to decrease by a similar amount as recollection estimates after a one-week delay. This is because the familiarity estimate formula also accounts for recollection estimates and these are mutually exclusive processes. Therefore, according to Yonelinas \& Jacoby's independence remember/know method (1995), Familiarity = "know responses"/(1 - Recollection) $($ Yonelinas, 2002). As such, should it have been possible for familiarity to have been estimated in this study, it is likely that similar decreases would have resulted. However, as seen in the studies reviewed by Yonelinas (2002), the decrease seen may have been sharper for recollection estimates than familiarity estimates, given that familiarity tends to be a gist-based process that, in general, is less sensitive than recollection is to forgetting (Yonelinas, 2002). In summary, the EEM effect was replicated in this study, such that emotional images were assigned more correct remember responses than neutral images, but this effect decreased following the one-week delay. Additionally, the emotion regulation task instructions also played a role in memory outcomes.

\section{Influence of Emotion Regulation Task Instructions on Memory}

Emotion regulation task instructions differentially affected correct remember responses. Importantly, this differentiation was observed despite not instructing participants on how to regulate their emotions, as was done in previous studies of emotion regulation (e.g., Dillon et al., 2007; Sheppes et al., 2011). More specifically, participants assigned more correct remember 
responses to images that had been paired with the instruction to enhance at encoding, regardless of image valence, than to the images that were paired with the suppress or maintain instruction. However, this expected effect was only present for images tested on the first test day. These results are largely congruent with the results from the emotion regulation and memory study by Dillon and colleagues (2007). However, one notable difference between these two studies is that Dillon and colleagues instructed participants to use a personal relevance strategy (i.e., to increase the personal relevance of the situation seen in the image) while viewing images paired with the enhance instruction, whereas in the current research, participants were not instructed to use any specific strategies while enhancing their emotional responses. Because this enhance effect was replicated in this study, regardless of the emotion regulation strategy used by participants at the time of encoding, it may be the general act of enhancing emotion, rather than the specific method by which the emotion is enhanced, that influences recognition memory for these images. Furthermore, in their study using a similar EMS task, Dillon and colleagues (2007) determined that images that were paired with the instruction to enhance were rated as more negative and more arousing than those in the maintain and suppress instruction conditions, even though the images were previously equated on these variables for each condition. Thus, the enhance condition in the current study may also have boosted recollection by increasing the emotional salience of the images; in future studies, it may be valuable to gain secondary ratings of participants' subjective view of the valence and arousal of the images.

With regards to images paired with the suppress instruction, it was predicted that the use of cognitive reappraisal strategies to suppress emotional responses would be positively correlated with the number of correct remember responses seen for these images, and that these responses would reach levels similar to those of the images paired with the enhance instruction. 
Conversely, it was predicted that if an autonomic or other idiosyncratic strategy was used to suppress one's emotional responses to the images, memory performance would be impaired, and would result in fewer correct remember responses than those images paired with the maintain instruction. This is because stimuli that are cognitively reappraised are thought to be more deeply encoded (Gross, 2001) than those that are responded to using autonomic methods. However, given that the percentage use of reappraisal, autonomic, or other emotion regulation strategies used did not influence memory for images paired with the suppress instruction, it was not statistically meaningful to parse out the differential effects of strategies used on the memory outcomes for images paired with the suppress instruction. Indeed, despite expectations that it may be possible to examine strategy use as a between-subjects variable (i.e., separate individuals into "groups" of strategy users, such as a group of "reappraisers"), this was not the case given that most participants used multiple strategies. Moreover, the discrepancies seen between the initial predictions and the results may reflect the fact that the predictions were based on the study by Dillon and colleagues (2007), who had directly compared memory for images paired with the instruction to decrease the personal relevance of an image with memory for images paired with the instruction to maintain a neutral facial expression when asked to suppress one's emotional responses to an image. As such, Dillon and colleagues' (2007) use of emotion regulation strategy instructions to ensure homogeneity in their sample's strategy use may have resulted in a clearer pattern of results favouring cognitive reappraisal over autonomic strategies than those seen in the study at hand. Therefore, it may be that by allowing participants the freedom to use these strategies may have inadvertently decreased the ability to assess differential effects of emotion regulation strategy use on memory in the current study. 
Emotion regulation task instructions also influenced correct know responses. Images that were paired with the instruction to maintain one's emotional response to the image were more often correctly assigned a know response than those paired with the instruction to enhance or suppress one's emotional response to the image, regardless of image valence. Again, these patterns only emerged during the first day of testing. These results suggest that an instruction to maintain may differentially affect gist-based, semantic memory (Yonelinas, 2002), as compared to the instructions to enhance or suppress one's emotional responses. Indeed, several qualitative responses written on the emotion regulation strategy questionnaire suggest that participants focused on the image content, rather than reappraising or mentally creating an emotional context to the image, to maintain the emotional reaction they experienced when initially viewing the image.

\section{Emotion Regulation Strategy Use}

Emotion regulation strategy use was examined in three ways. Trait-like spontaneous strategy use was measured by examining the types and the summed percentage amounts of strategies used during the first block of the questionnaire; state-like overall strategy use was measured by examining the types and the summed percentage amounts of all strategies used throughout the experiment; and the total percentage of reappraisal strategies and total percentage of autonomic strategies used were summed in total scores, respectively. These scores were correlated with memory outcomes and questionnaire measures that examined use of emotion regulation strategies in daily life.

In congruence with results reported by Jackson and colleagues (2000), participants in this study reported using a higher percentage of cognitive reappraisal strategies as compared to autonomic or idiosyncratic emotion regulation strategies, both after the first block of the task and 
throughout the task overall. Moreover, participants tended to use similar strategies, regardless of task instruction; overall use of the strategies within one task instruction correlated highly with their use of the same strategies within another. Moreover, despite several opportunities to describe their own strategy use, few participants reported using idiosyncratic strategies during the task, which lends support to the notion that reappraisal strategies tend to be used more often than other methods when participants make conscious emotion regulation decisions.

Although the relative proportion of reappraisal to autonomic strategy use was expected, the fact that the self-reported percent of strategies used by participants to modulate their emotional responses did not appear to influence their memory was not expected. Indeed, though it was expected that participants who used a greater percentage of cognitive reappraisal strategies to enhance their emotions, either during the first block of 40 images (as a measure of trait-like, spontaneous emotion regulation strategy use) or throughout the entire experiment (which may reflect state-like emotion regulation strategy use), would have better memory accuracy than participants who used autonomic or other strategies, the summed percentage of each strategy used bore no relationship to memory outcomes. Moreover, in an examination of the three specific reappraisal strategies chosen as primary forms of reappraisal for the emotion regulation questionnaire (i.e., cognitively reappraising the future outcome of the situation seen in the image, the severity of the situation seen in the image, and the personal relevance of the image), as well as the two primary forms of autonomic strategies (i.e., changing facial expression, changing breathing or muscle tension), none of these strategies appeared to uniquely (or jointly) influence recognition memory for the images. Given that most of the sample used reappraisal strategies, and many used autonomic strategies in conjunction, one explanation for these findings may be 
that a clear pattern favouring reappraisal over autonomic or other strategies, as seen throughout the literature, was not statistically possible to elucidate.

\section{Limitations and Future Directions}

There are several potential limitations to the study that may explain why a clear pattern of results favouring specific emotion regulation strategy use was not elucidated. One of the main objectives of this study was to examine which strategies participants used to regulate their emotions when presented with negative images. However, research on emotion regulation choice by Sheppes and colleagues (2011) demonstrated that the types of strategies chosen may be contingent on the arousal level of the image, such that individuals tend to use autonomic suppression while viewing high-arousing negative images as compared to cognitive reappraisal strategies while viewing low-arousing negative images. Although this study examined strategy use more broadly and across various image contexts (i.e., low- and high-arousing scenes with several types of image content, such as animals, people, and objects), an examination of strategy use as a function of image arousal in this or a similar study could perhaps have resulted in clearer patterns of relations between strategy use and memory outcomes. Although it was expected that the spontaneous emotion regulation strategies used during the first block of images of the EMS task would correlate with measures of emotion regulation in daily life, these results were not seen in the study at hand. This may be because emotion regulation strategy use in the laboratory setting, with emotional stimuli that are not immediately personally relevant, is not a viable proxy for daily-life emotion regulation strategy use. The employment of ecological, momentary assessment tools to evaluate emotion regulation strategy use, such as the use of a smartphone application that could prompt participants to fill in an emotion regulation survey at random intervals, would provide a richer dataset upon which daily-life strategy use could be 
approximated. Furthermore, researchers could explore ways to assess emotion regulation strategy use and memory in the laboratory setting in a more ecologically valid way; for example, by using naturalistic observation of individuals in an emotionally-arousing scenario, or by asking participants to reflect on which strategies they had used during an emotional experience. By comparing a richer set of daily-life emotion regulation strategy data to those used in a laboratory setting in a memory study, researchers would gain increased confidence that the strategies initially used did (or did not) approximate those used in daily life (i.e., "trait" strategy use).

Future research could also explore the extent to which emotion regulation strategies influence accuracy and response bias, which would allow for a more nuanced analysis of recollection and familiarity estimates. More specifically, by examining false alarms at the level of emotion regulation task instructions, an examination of recollection and familiarity processes could be conducted, which may provide novel insights into these processes. Because this study employed a within-subjects design, with the encoding task determining one of the key factors of the study (e.g., emotion regulation instructions), it was not possible to examine false alarms at the level of task instructions (because it is not possible to have a false alarm for images paired with the instruction to "enhance," for instance). As such, by employing a between-subjects design with the three task instructions comprising three separate groups, or if both the EMS and $\mathrm{R} / \mathrm{K}$ tasks were conducted in blocks, such that series of images were sequentially presented with the same task instruction, false alarm analyses could be run to determine whether individuals tended to falsely assign remember or know responses for images that were thought to be paired with a specific instruction.

Future studies could examine how response time influences remember, know, and new response decisions for images that were encoded with emotion regulation instructions. For 
instance, much research on the influence of response speeding (i.e., the process by which participants are forced to respond within a set time interval) on recognition memory suggests that response speeding tends to result in decreased recollection estimates while familiarity estimates remain relatively intact (Koen, Aly, Wang, \& Yonelinas, 2014). By comparing a self-paced study to one in which responses were speeded, future research could determine whether emotion regulation task instructions differentially influence remember and know responses within these designs. For instance, it may be that the enhance instruction invites participants to more deeply encode these images than those paired with the suppress or maintain instructions, resulting in less recollective decay during a speeded response task. Furthermore, the time-dependent effects of emotional stimuli on memory could be explored using a diffusion model (Ratcliff, 1978) to determine whether specific emotion regulation strategies influence response and memory biases (Bowen, Spaniol, Patel, \& Voss, 2016). Evaluating response timing, whether it be via response speeding or reaction time analyses, or using a diffusion model to investigate how emotion regulation strategies influence memory processes, are two of several avenues by which future researchers could evaluate how emotion regulation influences memory.

Sample characteristics may have also impacted on the ability to detect clear patterns of results. Given that the sample in this study included primarily female, educated, and healthy undergraduate students with little variation in strategy use, nuanced differences in memory ability at the level of strategy use may be more difficult to detect. Moreover, future research examining emotion regulation and memory in a diverse sample might allow demographic characteristics, such as age, gender, ethnicity, and level of education, to serve as moderating variables that would allow researchers to explore how emotion regulation influences memory across the lifespan of individuals from diverse cultures. Lastly, because emotion regulation is a 
core tenet of mental wellbeing (Ochsner \& Gross, 2005), the study of the influence of emotion regulation on memory in diverse clinical populations is important.

\section{Conclusion}

This thesis examined how the spontaneous use of conscious emotion regulation strategies during the EMS task (Jackson et al., 2000) influenced incidental recognition memory (i.e., remember and know responses) for emotional images as measured by the $\mathrm{R} / \mathrm{K}$ procedure (Tulving, 1985) across both an immediate test and one-week delay. In congruence with study hypotheses, the EEM effect was replicated, such that negative images were better remembered than neutral images across both test days. Moreover, on the immediate test day only, the EMS task instructions influenced participants' reported remember and know responses, such than images paired with the instruction to enhance one's emotional responses were better recollected than images paired with the instruction to either maintain or suppress their emotions, and the images paired with the instruction to maintain were more often assigned a correct know response than images paired with the instruction to enhance or suppress one's emotional responses. Despite this, the study's main hypothesis, that there would be a relation between the types of emotion regulation strategies used at encoding and subsequent recognition memory for emotional images, was unsupported. These findings suggest that although instructions to regulate emotional responses to both negative and neutral images differentially affect recognition memory in a highly educated undergraduate sample, more research is needed to determine whether specific emotion regulation strategies influence different memory processes. 
Appendix A: Demographic and Screening Interview (self-created, 2017)

Now I'd like to ask you some questions about you and your background. These questions will be stored securely and separately from your identifying information (consent form) and will only be linked through an arbitrary code \#. I remind you that your participation is voluntary, so you have the right to answer as many or as few of these questions as you wish.

1. Code \#

2. How old are you?

3. Sex: F / M / Other: / Prefer not to say

4. Is English your first language? $\mathrm{Y} / \mathrm{N}$

If no: At what age did you learn English?

Do you speak English fluently now? Y / N

5. Do you have normal or corrected-to-normal vision (e.g., wear glasses)?

$\mathrm{Y} / \mathrm{N}$

6. Do you have normal or corrected-to-normal hearing (e.g., use a hearing aid)? Y / N

7. Have you ever been diagnosed with a learning disability? $\mathrm{Y} / \mathrm{N}$

8. Have you ever lost consciousness (passed out or blacked out)? Y / N

If yes: How long did it last?

9. Have you ever been diagnosed with a neurological condition (e.g., seizures, traumatic brain injury, dementia, etc.)? $\mathrm{Y} / \mathrm{N}$

10. Are you currently taking any medications to treat/help with mental health issues (e.g., antidepressants, anti-anxiety medications, etc.)? Y / N

10. Have you ever been diagnosed with a mental health disorder? Y / N 
Appendix B: Emotion Regulation Verbal Instructions Administered during the Encoding Phase

\section{(Adapted from Jackson et al., 2000)}

While watching each picture, you will be instructed to either suppress, enhance, or maintain the emotion you are currently feeling in response to the picture. Suppose the emotion you are feeling in response to a picture is fear. Whatever fear you might experience in response to the picture, if you are instructed to ENHANCE, we would like you to increase the intensity of fear you feel. If you are instructed to SUPPRESS, we would like you to decrease the intensity of fear you feel. Similarly, if the emotion you experience in response to a picture is disgust and you are instructed to ENHANCE, we would like you to increase the intensity of disgust you feel. If you are instructed to SUPPRESS, we would like you to decrease the intensity of disgust you feel.

The other type of instruction you may receive is to LOOK. This is a cue that you should maintain the emotion you are feeling in response to a picture. For example, if you are feeling a certain kind of fear in response to a picture, and you are instructed to LOOK, we would like you to keep your fear at about that level. Similarly, if the emotion you are feeling in response to a picture is disgust, and you are instructed to LOOK, we would like you to keep your disgust at about the same level. Do you have any questions?

When suppressing, enhancing, or maintaining emotion you should stay focused on the picture and on the emotion you are feeling in response to the picture. For example, if you are feeling disgust in response to a picture and you are told to suppress your emotion, you should not accomplish this by generating a different emotion.

Try to concentrate on suppressing, enhancing, or maintaining your emotional reaction to each picture even after the picture disappears from the screen. A few seconds after the picture has disappeared, you will see a picture telling you to "RELAX." This is your cue to stop suppressing, enhancing, or maintaining your emotional response to the previous picture, and to get ready for the next picture.

Through the task, there will be 2 breaks at which time you will be asked to fill out a questionnaire on the computer, as well as a third questionnaire after the task is completed. This questionnaire will ask you to explain how you were suppressing, enhancing, or maintaining your emotion and will ask you to provide some examples of how you used certain strategies to enhance, maintain, or suppress your emotion. Do you have any questions about the questionnaire?

We are now ready to begin the practice trial.

[Practice trial is administered.]

During the practice trial, you were asked to enhance, maintain, and suppress your emotions to the images on your screen. Can you explain how you enhanced your emotion to the first image? Can you explain how you maintained your emotion to the second image? Can you explain how you suppressed your emotion to the third image?

We are now ready to begin the experiment. The experiment will consist of 6 blocks of pictures that last approximately 7 minutes each, with the surveys taking about 5 minutes each after the second, fourth, and sixth blocks. Overall, this will take approximately 60 minutes. Do you have any final questions?

You may now begin.

[Experimental task is administered.] 
Appendix C: Emotion Regulation Strategy Questionnaire (self-created, 2017)

The following questionnaire will assess what strategies you used to regulate your emotions when presented with the images and the different instruction words (e.g., Enhance, Look, or Suppress). For the purposes of the questionnaire, we are only interested in the emotion regulation strategies you used when presented with negative images.

You will be asked to complete this questionnaire three times throughout the experiment.

\section{Enhance}

1. What strategy or strategies did you use when you were asked to ENHANCE your emotions? Please select all strategies that you used. You will be asked to describe the strategy/strategies that you used on the next page.

a. I changed the meaning of the image by imagining that the negative situation depicted in the image was more severe than the image initially suggested.

For example, if I saw a picture of a hurt person, I imagined that the person was more badly hurt than shown in the image.

b. I changed the meaning of the image by imagining a more negative future outcome for the person or situation seen in the image.

For example, if I saw a picture of a hurt person, I imagined that the person would soon be injured further.

c. I changed the meaning of the image by imagining I was personally affected by the person or situation seen in the image.

For example, if I saw a picture of a hurt person, I imagined it was either myself or a person I cared about to enhance my emotions.

d. I changed my facial expression in response to the image to enhance my emotions.

For example, if I saw a picture of a hurt person, I frowned more strongly to enhance my emotions.

e. I changed my breathing or other bodily response (e.g., tensing muscles) to the image to enhance my emotions.

For example, if I saw a picture of a hurt person, I quickened my breathing or clenched my fists to enhance my emotions.

f. I used a strategy that was not listed to enhance my emotions. 
g. I am not able to identify which strategy/strategies I used.

h. I did not attempt to enhance my emotions.

i. I prefer not to say which strategy/strategies I used.

*Page break*

- You indicated that "I changed the meaning of the image by imagining that the negative situation depicted in the image was more severe than the image initially suggested." Please briefly describe (1-2 sentences) how you used this strategy when enhancing your emotions:

What percent of the time did you use this strategy when enhancing your emotions?

- You indicated that "I changed the meaning of the image by imagining a negative future outcome for the person or situation seen in the image." Please briefly describe (1-2 sentences) how you used this strategy when enhancing your emotions:

- What percent of the time did you use this strategy when enhancing your emotions?

- You indicated that "I changed the meaning of the image by imagining I was personally affected by the person or situation seen in the image." Please briefly describe (1-2 sentences) how you used this strategy when enhancing your emotions:

- What percent of the time did you use this strategy when enhancing your emotions?

- You indicated that "I changed my facial expression in response to the image to enhance my emotions." Please briefly describe (1-2 sentences) how you used this strategy when enhancing your emotions:

$\circ$ What percent of the time did you use this strategy when enhancing your emotions?

- You indicated that "I changed my breathing or other bodily response (e.g., tensing muscles) to the image to enhance my emotions." Please briefly describe (1-2 sentences) how you used this strategy when enhancing your emotions:

- What percent of the time did you use this strategy when enhancing your emotions? 
- You indicated that "I used a strategy that was not listed to enhance my emotions." Please describe this strategy (1-2 sentences) and how it helped you enhance your emotions:

- What percent of the time did you use this strategy when enhancing your emotions?

2. How successful were you at enhancing your emotions when using these strategies?
a. $0-$ Not at all
b. $1-$ A little bit
c. 2 - Somewhat
d. 3-Quite a bit
e. $4-\mathrm{A}$ great deal

3. On average, how much attention did you pay to each image when it was presented with the instruction to ENHANCE?
a. $0-$ Not at all
b. $1-$ A little bit
c. 2-Somewhat
d. 3-Quite a bit
e. $4-$ A great deal

\section{Suppress}

1. What strategy or strategies did you use when you were asked to SUPPRESS your emotions? Please select all strategies that you used. You will be asked to describe the strategy/strategies that you used on the next page.

a. I changed the meaning of the image by imagining that the negative situation depicted in the image was less severe than the image initially suggested.

For example, if I saw a picture of a hurt person, I imagined that the person was less badly hurt than shown in the image.

b. I changed the meaning of the image by imagining a positive future outcome for the person or situation seen in the image.

For example, if I saw a picture of a hurt person, I imagined that the person would soon be healed.

c. I changed the meaning of the image by imagining that I was not personally affected by the person or situation seen in the image. 
For example, if I saw a picture of a hurt person, I imagined that I was unaffected by their misfortune.

d. I changed my facial expression in response to the image to suppress my emotions.

For example, if I saw a picture of a hurt person, I tried not to show any emotion on my face.

e. I changed my breathing or other bodily response (e.g., tensing muscles) to the image to suppress my emotions.

For example, if I saw a picture of a hurt person, I slowed my breathing or relaxed my body to suppress my emotions.

f. I used a strategy that was not listed to suppress my emotions.

g. I am not able to identify which strategy/strategies I used when asked to suppress my emotions.

h. I did not attempt to suppress my emotions.

i. I prefer not to say which strategy/strategies I used.

*Page break*

- You indicated that "I changed the meaning of the image by imagining that the negative situation depicted in the image was less severe than the image initially suggested." Please briefly describe (1-2 sentences) how you used this strategy when suppressing your emotions:

- What percent of the time did you use this strategy when suppressing your emotions?

- You indicated that "I changed the meaning of the image by imagining a more positive future outcome for the person or situation seen in the image." Please briefly describe (1-2 sentences) how you used this strategy when suppressing your emotions:

○ What percent of the time did you use this strategy when suppressing your emotions?

- You indicated that "I changed the meaning of the image by imagining that I was not personally affected by the person or situation seen in 
the image." Please briefly describe (1-2 sentences) how you used this strategy when suppressing your emotions:

$\circ$ What percent of the time did you use this strategy when suppressing your emotions?

- You indicated that "I changed my facial expression in response to the image to suppress my emotions." Please briefly describe (1-2 sentences) how you used this strategy when suppressing your emotions:

$\circ$ What percent of the time did you use this strategy when suppressing your emotions?

- You indicated that "I changed my breathing or other bodily response (e.g., tensing muscles) to the image to suppress my emotions." Please briefly describe (1-2 sentences) how you used this strategy when suppressing your emotions:

- What percent of the time did you use this strategy when suppressing your emotions?

- You indicated that "I used a strategy that was not listed to suppress my emotions." Please describe this strategy (1-2 sentences) and how it helped you suppress your emotions:

$\circ$ What percent of the time did you use this strategy when suppressing your emotions?

2. How successful were you at suppressing your emotions when using these strategies?
a. $0-$ Not at all
b. 1 - A little bit
c. 2-Somewhat
d. 3-Quite a bit
e. $4-\mathrm{A}$ great deal

3. On average, how much attention did you pay to each image when it was presented with an instruction to SUPPRESS your emotions?
a. 0-Not at all
b. 1 - A little bit
c. 2-Somewhat
d. 3-Quite a bit
e. 4-A great deal 


\section{Maintain}

1. What strategy or strategies did you use when you were asked to MAINTAIN your emotions? Please select all strategies that you used. You will be asked to describe the strategy/strategies that you used on the next page.

a. I changed the meaning of the image by imagining that the negative situation depicted in the image was either more or less severe than the image initially suggested.

For example, if I saw a picture of a hurt person, I imagined that the person was either more or less badly hurt than shown in the image, to maintain my emotional state.

b. I changed the meaning of the image by imagining a more or less negative future outcome for the person or situation seen in the image.

For example, if I saw a picture of a hurt person, I imagined that the person would soon be injured further or would be healed soon, to maintain my emotional state.

c. I changed the meaning of the image by imagining I was or was not personally affected by the person or situation seen in the image.

For example, if I saw a picture of a hurt person, I imagined it was either myself or a person I cared about, or I personally distanced myself, to maintain my emotional state.

d. I changed my facial expression in response to the image to maintain my emotions.

For example, if I saw a picture of a hurt person, I frowned more strongly, or smiled to maintain my emotional state.

e. I changed my breathing or other bodily response (e.g., tensing muscles) to the image to maintain my emotions.

For example, if I saw a picture of a hurt person, I quickened my breathing or clenched my fists, or did the opposite, to maintain my emotional state.

f. I used a strategy that was not listed to maintain my emotions.

g. I am not able to identify which strategy/strategies I used.

h. I did not attempt to maintain my emotions. 


\section{i. I prefer not to say which strategy/strategies I used.}

*Page break*

- You indicated that "I changed the meaning of the image by imagining that the negative situation depicted in the image was either more or less severe than the image initially suggested."

- Please briefly describe (1-2 sentences) how you used this strategy when maintaining your emotions:

- What percent of the time did you use this strategy when maintaining your emotions?

- You indicated that "I changed the meaning of the image by imagining a more or less negative future outcome for the person or situation seen in the image." Please briefly describe (1-2 sentences) how you used this strategy when maintaining your emotions:

- What percent of the time did you use this strategy when maintaining your emotions?

- You indicated that "I changed the meaning of the image by imagining I was or was not personally affected by the person or situation seen in the image."

- Please briefly describe (1-2 sentences) how you used this strategy when maintaining your emotions:

$\circ$ What percent of the time did you use this strategy when maintaining your emotions?

- You indicated that "I changed my facial expression in response to the image to maintain my emotions."

- Please briefly describe (1-2 sentences) how you used this strategy when maintaining your emotions:

- What percent of the time did you use this strategy when maintaining your emotions?

- You indicated that "I changed my breathing or other bodily response (e.g., tensing muscles) to the image to maintain my emotions." Please briefly describe (1-2 sentences) how you used this strategy when maintaining your emotions:

- What percent of the time did you use this strategy when maintaining your emotions? 
- You indicated that "I used a strategy that was not listed to maintain my emotions." Please describe this strategy (1-2 sentences) and how it helped you maintain your emotions:

$\circ$ What percent of the time did you use this strategy when maintaining your emotions?

2. How successful were you at maintaining your emotions when using these strategies?
a. 0 - Not at all
b. $1-$ A little bit
c. 2 - Somewhat
d. 3-Quite a bit
e. 4-A great deal

3. On average, how much attention did you pay to each image when it was presented with an instruction to MAINTAIN your emotions?
f. $0-$ Not at all
g. 1-A little bit
h. 2-Somewhat
i. 3-Quite a bit
j. $4-$ A great deal 


\section{Supplementary Questions for Questionnaires 2 and 3}

\section{Enhance}

Did the strategies that you use when asked to ENHANCE your emotions change from the last time we asked you?
a. Yes
b. No
c. Unsure

- If yes, why did they change?:

\section{Suppress}

Did the strategies that you use when asked to SUPPRESS your emotions change from the last time we asked you?
a. Yes
b. No
c. Unsure

- If yes, why did they change?:

\section{Maintain}

Did the strategies that you use when asked to MAINTAIN your emotions change from the last time we asked you?
a. Yes
b. No
c. Unsure

- If yes, why did they change?: 
Appendix D: Computerized Instructions for the Remember/Know Task (Adapted from Patel, 2009)

Slide 1: In the first part of this experiment, you saw a series of images. This was called the "study phase". You will now be shown some of the images you saw in the study phase along with new images. You will be asked which images are new and which ones you recognize from the study phase...

Slide 2: There are different kinds of recognition. For instance, recognition can bring back to mind something you recollect about what it is that you recognize. For example, you might recognize someone's face and REMEMBER thinking that person had similar eyes to yours. At other times, recognition brings nothing back to mind about what it is you recognize. As when, for example, you are confident that you recognize someone, and you KNOW you recognize them, because of strong feelings of familiarity - but you have no recollection of seeing this person before. You do not recall anything specific about them.

Slide 3: The purpose of this part of the experiment is to look at how these different types of recognition apply to the images that you saw earlier today in the study phase. I will briefly describe these now and then we will practice a few examples together to confirm that the instructions are clear.

Sometimes when you recognize an image as one you saw in the study phase, recognition will bring back to mind something you REMEMBER thinking about when the image appeared then. You recollect something you consciously experienced at that time.

Other times recognizing an image as one you saw in the study phase will not bring back to mind anything specific you remember about seeing it then. Instead, the image will seem familiar, so that you feel confident and KNOW that it was one you saw in the study phase, even though you do not recollect anything you experienced when you saw it then.

Slide 4: You will be asked to indicate whether the image being shown to you is:

"NEW" (You did not see it in the study phase)

"REMEMBER" (You saw it in the study phase and can recall how you felt or what you thought when you were viewing the image, or any other experiences you had while viewing the image) "KNOW" (You are aware that you must have seen it in the study phase, but you cannot explicitly remember viewing it)

Slide 5: So just to recap, you will answer "NEW" if you did not see the image in the study phase.

You will answer "REMEMBER" if recognition is accompanied by some specific or vivid recollective experience.

Or you will answer "KNOW" if you recognize an image with strong feelings of familiarity, but without any specific recollective experience. 
[Proceed to verbal instructions]

\section{Supplementary Oral Instructions to Computerized Instructions}

This can be a tricky task to keep straight because of all the types of responses. So although you've just read the instructions, I'm going to now go over them with you again and show how things will look on the computer.

You will see the images one at a time. If you do not recognize the image as previously being in the Emotion Regulation Task then you press $\mathbf{N}$ for "New". If you recognize an image, we would like to know how well you remember it. If it triggers something that you experienced when you saw it previously, like, for example, something about its appearance on the screen or the order in which the image came in, then I would like you to indicate this kind of recognition by pressing $\mathbf{R}$ for Remember. In other instances the image may remind you of something you thought about when you saw it previously, like it reminded you of a friend or family member, or an image that you formed when you saw the image, or something of personal significance that you associated with the image. Again, if you can recollect any of these aspects of when the image was presented, then I would like you to press $\mathbf{R}$ for Remember.

Do you have any questions about Remember responses? Can you give me an example of something that you remember? (confirm or clarify understanding as necessary)

At other times you will see an image and you will recognize it as one that you saw in the Emotion Regulation Task, but the image will not bring back to mind anything you remember about seeing it then, the image will just seem extremely familiar. When you feel confident that you saw the image, even though you do not recollect anything you experienced when you saw it, I would like you to indicate this kind of recognition, by pressing $\mathbf{K}$ for Know. With "Know" responses you are sure about seeing the image in the Emotion Regulation Task but cannot remember the circumstances in which the image was presented, or the thoughts elicited by the image. Do you have any questions about Know responses?

We are now ready to practice. Make sure to say your response of Remember, Know, or New out loud. 


\section{Appendix E: False Alarm Analysis}

The influence of the emotion regulation task on remember and know false alarm rates (i.e., new images that were incorrectly assigned a "remember" or "know" response) was analyzed via a 2 (Day: 1,2$) \times 2($ Response: Remember, Know $) \times 2($ Valence: Negative, Neutral $)$ factorial within-subjects ANOVA on the sample of 58 participants who completed both test days. The resulting $2 \times 2 \times 2$ ANOVA revealed significant a main effect of Day, $F(1,57)=12.27, p<$ $.001, \eta_{p}{ }^{2}=.18$, such that there were more false alarms on Day 2 than Day 1 . The ANOVA also revealed a significant main effect of Response, $F(1,57)=66.46, p<.001, \eta_{p}{ }^{2}=.54$, such that there were more false alarms for know responses than remember responses. No significant main effect of Valence was revealed, $F(1,57)=.32, p=.576, \eta_{p}^{2}=.09$

The main effect of Day was qualified by a significant Day by Valence interaction, $F(1$, $57)=48.28, p<.001, \eta_{p}{ }^{2}=.46$. Simple effects analyses revealed that on day 1 of testing, there were more negative than neutral false alarms, mean difference $=.04, S E<.01, p<.001$. By contrast, on Day 2 of testing, there was no significant difference between the valence of images that were incorrectly assigned a remember or know response, mean difference $>-0.01, S E<.01$, $p=.415$ (See Figure E1). 


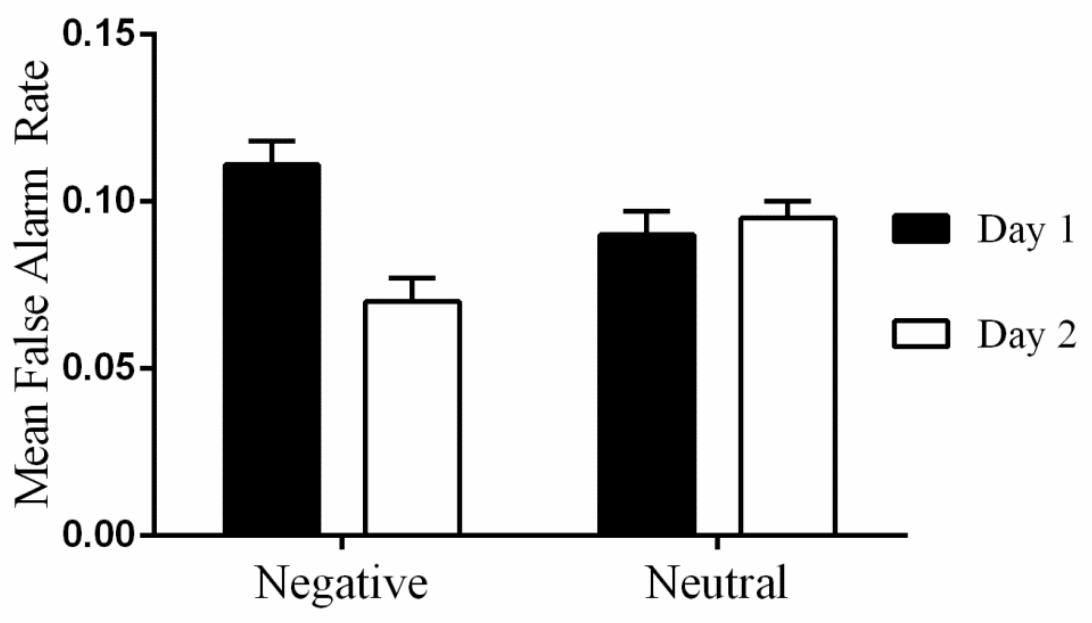

Figure E1. Mean false alarm rate values on both test days, for both negative and neutral images $($ error bars $=\mathrm{SEM})$.

No other interaction was significant: Day $\times$ Response, $F(1,57)=.904, p=.346, \eta p^{2}$ $=.016$; Valence $\times$ Response, $F(1,57)=.004, p=.949, \eta p^{2}<.001$; Day $\times$ Valence $\times$ Response, $F(1,57)=1.268, p=.265, \eta p^{2}=.022$. 
Appendix F: Correlational Analyses

Table F1

Correlations Between Spontaneous Emotion Regulation Strategy Use for Enhance and Remember and Know Responses for Images Paired with the Instruction to Enhance

\begin{tabular}{|c|c|c|c|c|}
\hline & $\begin{array}{c}\text { Day } 1 \\
\text { Remember }\end{array}$ & Day 1 Know & $\begin{array}{c}\text { Day } 2 \\
\text { Remember }\end{array}$ & Day 2 Know \\
\hline Severity & .041 & -.165 & .012 & -.124 \\
\hline Future & .050 & .028 & .065 & .202 \\
\hline Outcome & & & & \\
\hline Personal & -.238 & .250 & -.212 & .211 \\
\hline Relevance & & & & \\
\hline $\begin{array}{c}\text { Facial } \\
\text { Expression }\end{array}$ & -.008 & .035 & .013 & -.089 \\
\hline Breathing & .125 & -.105 & -.238 & .036 \\
\hline Other & .131 & -.021 & .114 & .121 \\
\hline
\end{tabular}

Table F2

Correlations Between Overall Emotion Regulation Strategy Use for Enhance and Remember and Know Responses for Images Paired with the Instruction to Enhance

\begin{tabular}{cllll}
\hline & \multicolumn{1}{c}{$\begin{array}{c}\text { Day 1 } \\
\text { Remember }\end{array}$} & Day 1 Know & $\begin{array}{c}\text { Day 2 } \\
\text { Remember }\end{array}$ & Day 2 Know \\
\hline Severity & .035 & -.162 & .186 & $-.323^{\mathrm{a}}$ \\
Future & .002 & .099 & .067 & .235 \\
$\begin{array}{c}\text { Outcome } \\
\text { Personal }\end{array}$ & -.159 & .123 & -.205 & .160 \\
$\begin{array}{c}\text { Relevance } \\
\text { Facial }\end{array}$ & .075 & .001 & -.056 & .136 \\
$\begin{array}{c}\text { Expression } \\
\text { Breathing }\end{array}$ & .043 & .002 & -.204 & .223 \\
Other & .012 & .061 & -.056 & .204 \\
\hline${ }^{\mathrm{p}=<0.05}$ & & & &
\end{tabular}


Table F3

Correlations Between Spontaneous Emotion Regulation Strategy Use for Suppress and Remember and Know Responses for Images Paired with the Instruction to Suppress

\begin{tabular}{|c|c|c|c|c|}
\hline & $\begin{array}{c}\text { Day } 1 \\
\text { Remember }\end{array}$ & Day 1 Know & $\begin{array}{c}\text { Day } 2 \\
\text { Remember }\end{array}$ & Day 2 Know \\
\hline Severity & .083 & -.070 & -.031 & -.131 \\
\hline Future & .132 & -.058 & $.329^{a}$ & .012 \\
\hline Outcome & & & & \\
\hline Personal & -.165 & .175 & -.146 & .010 \\
\hline Relevance & & & & \\
\hline Facial & -.065 & .113 & .032 & .062 \\
\hline Expression & & & & \\
\hline Breathing & .146 & -.069 & .053 & .103 \\
\hline Other & .117 & -.169 & .025 & .174 \\
\hline
\end{tabular}

Table F4

Correlations Between Overall Emotion Regulation Strategy Use for Suppress and Remember and Know Responses for Images Paired with the Instruction to Suppress

\begin{tabular}{|c|c|c|c|c|}
\hline & $\begin{array}{c}\text { Day } 1 \\
\text { Remember }\end{array}$ & Day 1 Know & $\begin{array}{c}\text { Day } 2 \\
\text { Remember }\end{array}$ & Day 2 Know \\
\hline Severity & .157 & -.171 & .150 & -.039 \\
\hline Future & .128 & -.061 & .077 & .180 \\
\hline Outcome & & & & \\
\hline $\begin{array}{l}\text { Personal } \\
\text { Relevance }\end{array}$ & -.014 & .094 & -.047 & .138 \\
\hline $\begin{array}{c}\text { Facial } \\
\text { Expression }\end{array}$ & .143 & -.126 & -.048 & .228 \\
\hline Breathing & -.067 & .076 & .007 & .040 \\
\hline Other & .103 & -.069 & -.142 & .101 \\
\hline
\end{tabular}


Table F5

Correlations Between Daily-Life Emotion Regulation Strategy Use for Enhance and Remember and Know Responses for Images Paired with the Instruction to Enhance Day 1 Day 1 Know Day 2 Day 2 Know Remember Remember

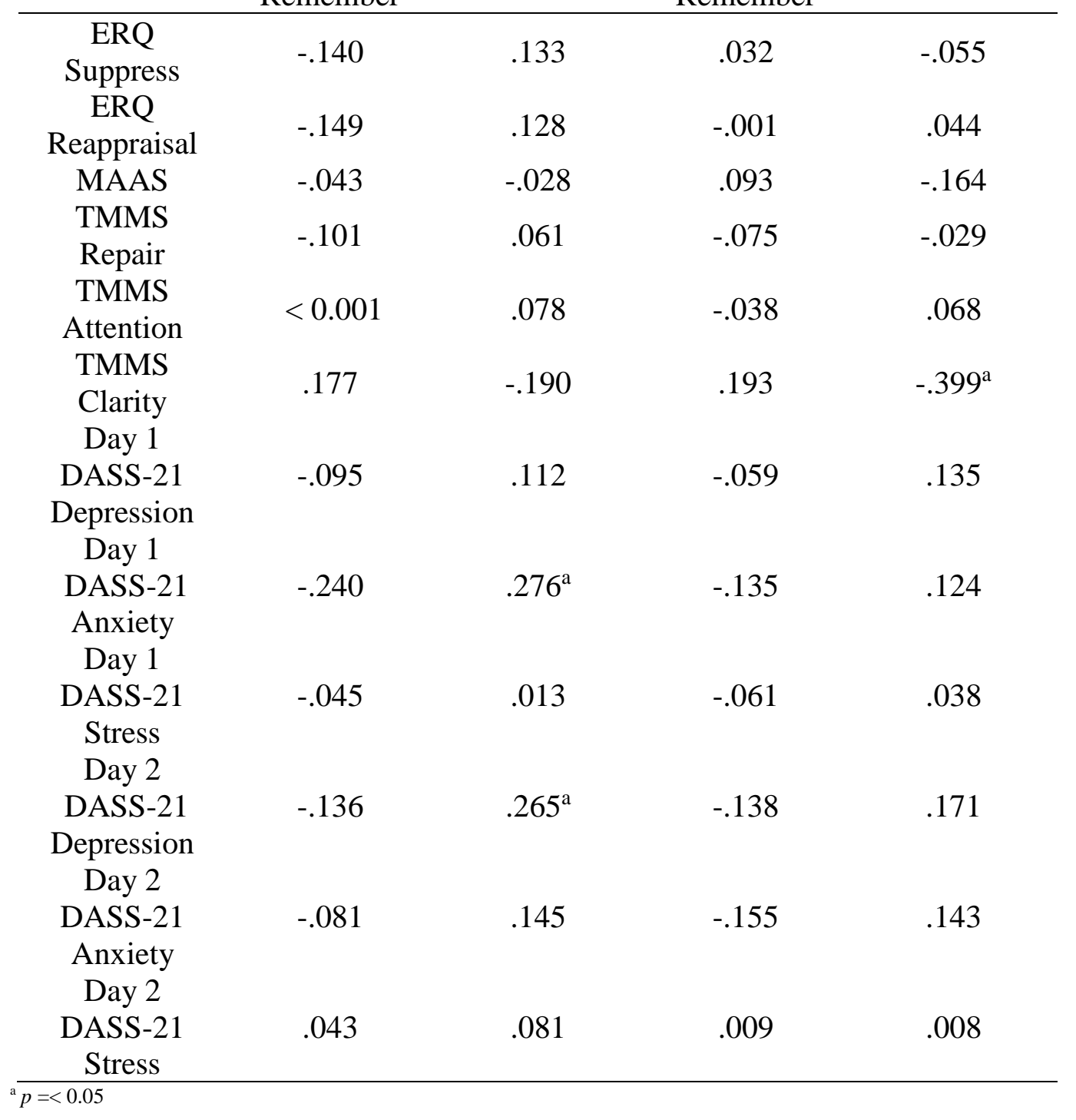


Table F6

Correlations Between Daily-Life Emotion Regulation Strategy Use for Suppress and Remember and Know Responses for Images Paired with the Instruction to Suppress

Day 1 Day 1 Know Day 2 Day 2 Know
Remember Remember

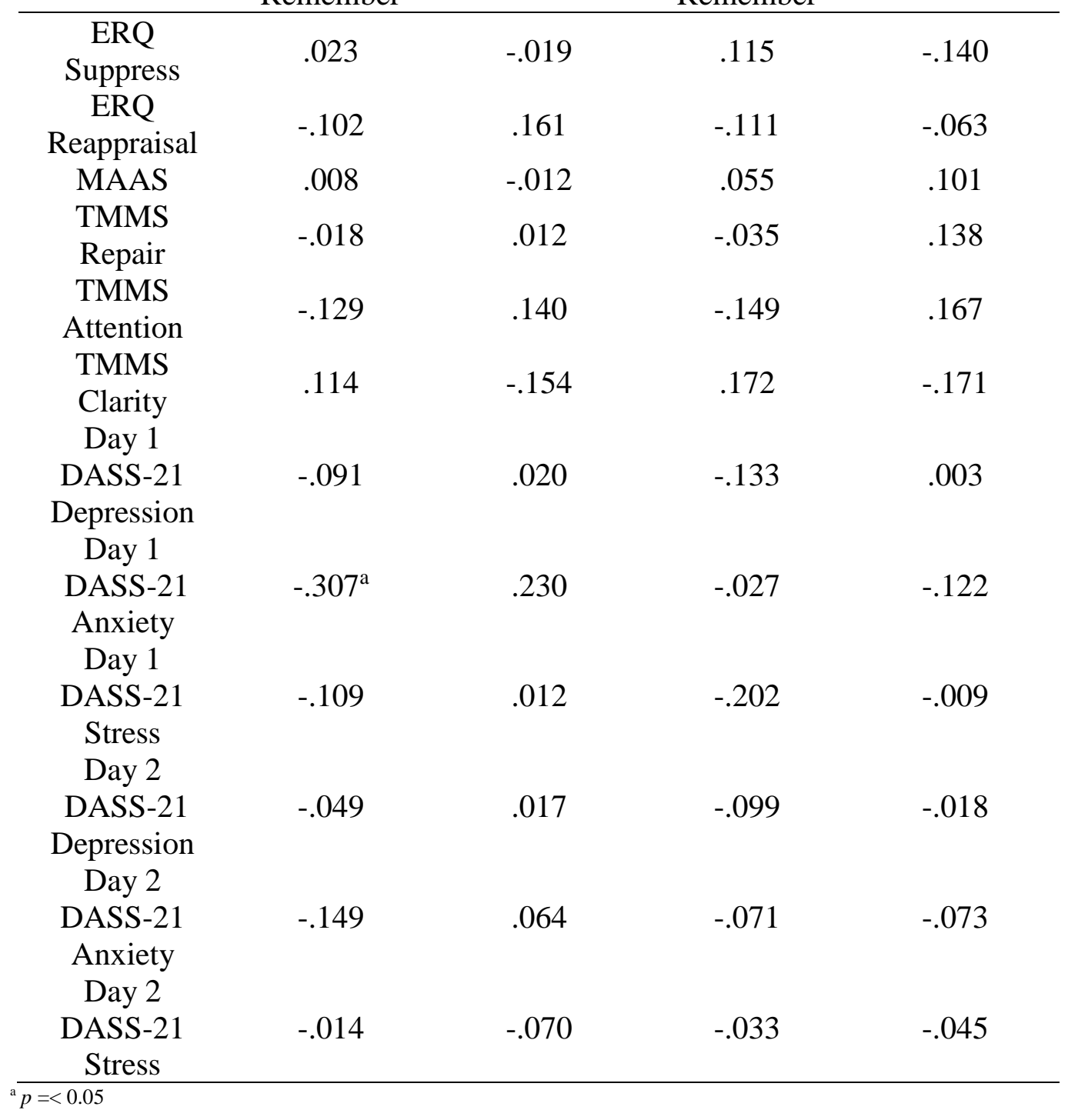


Table F7

Correlations Between Daily-Life Emotion Regulation Strategy Use for Enhance and Percentage Spontaneous Emotion Regulation Strategy Use for Images Paired with the Instruction to Enhance

\begin{tabular}{|c|c|c|c|c|c|c|}
\hline & Severity & $\begin{array}{c}\text { Future } \\
\text { Outcome }\end{array}$ & $\begin{array}{c}\text { Personal } \\
\text { Relevance }\end{array}$ & $\begin{array}{c}\text { Facial } \\
\text { Expression }\end{array}$ & Breathing & Other \\
\hline $\begin{array}{c}\text { ERQ } \\
\text { Suppress }\end{array}$ & .005 & .050 & .013 & .076 & .042 & .118 \\
\hline $\begin{array}{c}\text { ERQ } \\
\text { Reappraisal }\end{array}$ & -.095 & .156 & .163 & -.078 & -.014 & -.084 \\
\hline MAAS & .022 & -.043 & -.186 & .087 & -.038 & .108 \\
\hline $\begin{array}{l}\text { TMMS } \\
\text { Repair }\end{array}$ & .029 & -.096 & .092 & .055 & -.171 & -.002 \\
\hline $\begin{array}{l}\text { TMMS } \\
\text { Attention }\end{array}$ & .079 & .062 & .198 & .200 & -.146 & .098 \\
\hline $\begin{array}{l}\text { TMMS } \\
\text { Clarity } \\
\text { Day } 1\end{array}$ & .140 & -.109 & $-.261^{\mathrm{a}}$ & .094 & -.133 & .017 \\
\hline $\begin{array}{c}\text { DASS-21 } \\
\text { Depression } \\
\text { Day } 1\end{array}$ & -.060 & -.076 & .183 & .116 & .058 & .052 \\
\hline $\begin{array}{l}\text { DASS-21 } \\
\text { Anxiety } \\
\text { Day } 1\end{array}$ & $-.259^{a}$ & -.071 & $.389^{\mathrm{a}}$ & -.012 & .184 & -.087 \\
\hline $\begin{array}{c}\text { DASS-21 } \\
\text { Stress } \\
\text { Day } 2\end{array}$ & -.073 & -.112 & $.264^{\mathrm{a}}$ & .019 & -.075 & -.122 \\
\hline $\begin{array}{c}\text { DASS-21 } \\
\text { Depression } \\
\text { Day } 2\end{array}$ & -.065 & .078 & .172 & .158 & .154 & -.015 \\
\hline $\begin{array}{c}\text { DASS-21 } \\
\text { Anxiety } \\
\text { Day } 2\end{array}$ & -.159 & -.051 & .153 & .037 & .246 & -.013 \\
\hline $\begin{array}{l}\text { DASS-21 } \\
\text { Stress }\end{array}$ & -.054 & .109 & -.029 & .099 & .158 & -.037 \\
\hline
\end{tabular}


Table F8

Correlations Between Daily-Life Emotion Regulation Strategy Use for Enhance and Percentage Overall Emotion Regulation Strategy Use for Images Paired with the Instruction to Enhance

\begin{tabular}{|c|c|c|c|c|c|c|c|c|}
\hline & $\begin{array}{c}\text { Severit } \\
\mathrm{y}\end{array}$ & $\begin{array}{c}\text { Future } \\
\text { Outco } \\
\text { me }\end{array}$ & $\begin{array}{c}\text { Personal } \\
\text { Relevan } \\
\text { ce }\end{array}$ & $\begin{array}{c}\text { Overall } \\
\text { Reapprais } \\
\text { al }\end{array}$ & $\begin{array}{c}\text { Facial } \\
\text { Expressi } \\
\text { on }\end{array}$ & $\begin{array}{c}\text { Breathi } \\
\text { ng }\end{array}$ & $\begin{array}{l}\text { Overall } \\
\text { Autonom } \\
\text { ic }\end{array}$ & $\begin{array}{c}\text { Overa } \\
11 \\
\text { Other }\end{array}$ \\
\hline $\begin{array}{c}\text { ERQ } \\
\text { Suppress } \\
\text { ERQ }\end{array}$ & .039 & .063 & -.056 & .014 & -.046 & .136 & .060 & .115 \\
\hline $\begin{array}{c}\text { Reapprais } \\
\text { al }\end{array}$ & .003 & .159 & -.121 & -.029 & -.189 & -.195 & -.234 & -.142 \\
\hline MAAS & .161 & .005 & -.120 & .054 & .013 & -.186 & -.111 & .183 \\
\hline $\begin{array}{l}\text { TMMS } \\
\text { Repair }\end{array}$ & .049 & -.077 & -.008 & -.006 & -.209 & $-.259^{\mathrm{a}}$ & $-.286^{\mathrm{a}}$ & -.054 \\
\hline $\begin{array}{l}\text { TMMS } \\
\text { Attention }\end{array}$ & .044 & .068 & .035 & .081 & .179 & -.235 & -.046 & -.054 \\
\hline $\begin{array}{l}\text { TMMS } \\
\text { Clarity } \\
\text { Day } 1\end{array}$ & .138 & -.151 & -.168 & -.090 & -.083 & -.238 & -.200 & .042 \\
\hline $\begin{array}{c}\text { DASS-21 } \\
\text { Depressio } \\
n\end{array}$ & -.043 & -.005 & -.083 & -.080 & .087 & .141 & .140 & -.090 \\
\hline $\begin{array}{c}\text { Day 1 } \\
\text { DASS- } \\
21 \\
\text { Anxiety } \\
\text { Day } 1\end{array}$ & -.171 & -.080 & .080 & -.097 & .035 & $.294^{\mathrm{a}}$ & .207 & -.094 \\
\hline $\begin{array}{c}\text { DASS-21 } \\
\text { Stress }\end{array}$ & -.075 & -.067 & .137 & .020 & -.003 & .034 & .020 & -.087 \\
\hline $\begin{array}{c}\text { Day } 2 \\
\text { DASS- } \\
21\end{array}$ & -.134 & .133 & .145 & .054 & $.270^{\mathrm{a}}$ & .208 & $.289^{\mathrm{a}}$ & -.018 \\
\hline $\begin{array}{c}\text { Depressio } \\
\mathrm{n} \\
\text { Day } 2\end{array}$ & & & & & & & & \\
\hline $\begin{array}{c}\text { DASS-21 } \\
\text { Anxiety } \\
\text { Day } 2\end{array}$ & -.142 & -.003 & .087 & -.038 & .176 & $.406^{\mathrm{a}}$ & $.360^{\mathrm{a}}$ & -.119 \\
\hline $\begin{array}{c}\text { DASS-21 } \\
\text { Stress }\end{array}$ & -.016 & .190 & .093 & .114 & .225 & $.293^{\mathrm{a}}$ & $.317^{\mathrm{a}}$ & -.016 \\
\hline
\end{tabular}


Table F9

Correlations Between Daily-Life Emotion Regulation Strategy Use for Suppress and Percentage Spontaneous Emotion Regulation Strategy Use for Images Paired with the Instruction to

Suppress

\begin{tabular}{|c|c|c|c|c|c|c|}
\hline & Severity & $\begin{array}{c}\text { Future } \\
\text { Outcome }\end{array}$ & $\begin{array}{c}\text { Personal } \\
\text { Relevance }\end{array}$ & $\begin{array}{c}\text { Facial } \\
\text { Expression }\end{array}$ & Breathing & Other \\
\hline $\begin{array}{c}\text { ERQ } \\
\text { Suppress }\end{array}$ & $\begin{array}{c}-.343 p= \\
0.008\end{array}$ & .151 & .077 & .220 & .078 & -.226 \\
\hline $\begin{array}{c}\text { ERQ } \\
\text { Reappraisal }\end{array}$ & .204 & -.192 & .029 & -.111 & .009 & -.042 \\
\hline MAAS & .052 & -.011 & -.091 & .073 & -.091 & .125 \\
\hline $\begin{array}{l}\text { TMMS } \\
\text { Repair }\end{array}$ & .093 & -.026 & -.099 & -.140 & -.071 & -.148 \\
\hline $\begin{array}{c}\text { TMMS } \\
\text { Attention }\end{array}$ & .254 & -.059 & -.210 & $.266^{\mathrm{a}}$ & -.012 & .077 \\
\hline $\begin{array}{l}\text { TMMS } \\
\text { Clarity } \\
\text { Day } 1\end{array}$ & .204 & .141 & -.206 & -.088 & -.168 & -.152 \\
\hline $\begin{array}{c}\text { DASS-21 } \\
\text { Depression } \\
\text { Day } 1\end{array}$ & $-.273^{\mathrm{a}}$ & -.045 & .149 & $.270^{\mathrm{a}}$ & .177 & .039 \\
\hline $\begin{array}{l}\text { DASS-21 } \\
\text { Anxiety } \\
\text { Day } 1\end{array}$ & -.225 & -.127 & .033 & .174 & .215 & .052 \\
\hline $\begin{array}{l}\text { DASS-21 } \\
\text { Stress } \\
\text { Day } 2\end{array}$ & .015 & -.141 & -.010 & .077 & .056 & .150 \\
\hline $\begin{array}{c}\text { DASS-21 } \\
\text { Depression } \\
\text { Day } 2\end{array}$ & -.153 & -.028 & .039 & .179 & .177 & .047 \\
\hline $\begin{array}{l}\text { DASS-21 } \\
\text { Anxiety } \\
\text { Day } 2\end{array}$ & $-.283^{\mathrm{a}}$ & -.206 & .179 & .182 & .239 & -.057 \\
\hline $\begin{array}{l}\text { DASS-21 } \\
\text { Stress }\end{array}$ & -.032 & -.156 & .152 & .012 & .204 & .015 \\
\hline
\end{tabular}


Table F10

Correlations Between Daily-Life Emotion Regulation Strategy Use for Suppress and Percentage Overall Emotion Regulation Strategy Use for Images Paired with the Instruction to Suppress

\begin{tabular}{|c|c|c|c|c|c|c|c|c|}
\hline & $\begin{array}{c}\text { Severit } \\
y\end{array}$ & $\begin{array}{c}\text { Future } \\
\text { Outco } \\
\text { me }\end{array}$ & $\begin{array}{c}\text { Personal } \\
\text { Relevan } \\
\text { ce }\end{array}$ & $\begin{array}{c}\text { Overall } \\
\text { Reapprais } \\
\text { al }\end{array}$ & $\begin{array}{c}\text { Facial } \\
\text { Expressi } \\
\text { on }\end{array}$ & $\begin{array}{c}\text { Breathi } \\
\text { ng }\end{array}$ & $\begin{array}{l}\text { Overall } \\
\text { Autonom } \\
\text { ic }\end{array}$ & $\begin{array}{c}\text { Overa } \\
11 \\
\text { Other }\end{array}$ \\
\hline $\begin{array}{c}\text { ERQ } \\
\text { Suppress } \\
\text { ERQ }\end{array}$ & -.254 & . 197 & .031 & -.053 & .050 & .088 & .098 & -.159 \\
\hline $\begin{array}{c}\text { Reapprais } \\
\text { al }\end{array}$ & .091 & -.127 & .186 & .100 & -.235 & -.194 & -.292 & -.133 \\
\hline MAAS & .210 & .003 & -.076 & .118 & .093 & -.264 & -.143 & .121 \\
\hline $\begin{array}{l}\text { TMMS } \\
\text { Repair }\end{array}$ & .111 & -.092 & -.008 & .004 & -.204 & -.240 & $-.309^{a}$ & -.171 \\
\hline $\begin{array}{l}\text { TMMS } \\
\text { Attention }\end{array}$ & $.275^{\mathrm{a}}$ & -.028 & -.003 & .164 & . 168 & -.115 & .016 & .016 \\
\hline $\begin{array}{l}\text { TMMS } \\
\text { Clarity } \\
\text { Day } 1\end{array}$ & $.274^{\mathrm{a}}$ & -.003 & -.064 & .137 & -.050 & -.257 & -.227 & -.148 \\
\hline $\begin{array}{c}\text { DASS-21 } \\
\text { Depressio } \\
\mathrm{n}\end{array}$ & -.221 & -.022 & .022 & -.168 & .130 & .200 & .232 & -.017 \\
\hline $\begin{array}{c}\text { Day } 1 \\
\text { DASS- } \\
21\end{array}$ & -.153 & -.098 & -.137 & -.218 & -.146 & $.370^{\mathrm{a}}$ & 190 & .069 \\
\hline $\begin{array}{l}\text { Anxiety } \\
\text { Day } 1\end{array}$ & & & & & & & & \\
\hline $\begin{array}{c}\text { DASS-21 } \\
\text { Stress } \\
\text { Day } 2 \\
\text { DASS- }\end{array}$ & .047 & -.050 & -.234 & -.127 & -.059 & .189 & . 107 & .112 \\
\hline $\begin{array}{c}21 \\
\text { Depressio } \\
\mathrm{n} \\
\text { Day } 2\end{array}$ & -.092 & .018 & -.050 & -.081 & .154 & $.260^{\mathrm{a}}$ & $.293^{\mathrm{a}}$ & .057 \\
\hline $\begin{array}{c}\text { DASS-21 } \\
\text { Anxiety } \\
\text { Day } 2\end{array}$ & -.207 & $-.263^{\mathrm{a}}$ & .147 & -.214 & .087 & $.428^{\mathrm{a}}$ & $.379^{\mathrm{a}}$ & -.042 \\
\hline $\begin{array}{c}\text { DASS-21 } \\
\text { Stress } \\
\end{array}$ & .084 & -.119 & .180 & .070 & .087 & $.352^{\mathrm{a}}$ & $.322^{\mathrm{a}}$ & .043 \\
\hline
\end{tabular}




\section{References}

Arch, J. J., \& Craske, M. G. (2006). Mechanisms of mindfulness: Emotion regulation following a focused breathing induction. Behaviour Research and Therapy, 44, 1849-1858.

Bishop, S. R., Lau, M., Shapiro, S., Carlson, L., Anderson, N. D., Carmody, J., ... \& Devins, G. (2004). Mindfulness: A proposed operational definition. Clinical Psychology: Science and Practice, 11, 230-241.

Bonanno, G. A., Papa, A., Lalande, K., Westphal, M., \& Coifman, K. (2004). The importance of being flexible: The ability to both enhance and suppress emotional expression predicts long-term adjustment. Psychological Science, 15, 482-487.

Bowen, H. J., Spaniol, J., Patel, R., \& Voss, A. (2016). A diffusion model analysis of decision biases affecting delayed recognition of emotional stimuli. PLoS One, 11, e0146769.

Brown, K. W., \& Ryan, R. M. (2003). The benefits of being present: Mindfulness and its role in psychological well-being. Journal of Personality and Social Psychology, 84, 822.

Dillon, D. G., Ritchey, M., Johnson, B. D., \& LaBar, K. S. (2007). Dissociable effects of conscious emotion regulation strategies on explicit and implicit memory. Emotion, 7, 354-365.

Dunn, J. C. (2008). The dimensionality of the remember-know task: A state-trace analysis. Psychological Review, 115, 426-446.

Faul, F., Erdfelder, E., Lang, A.-G. \& Buchner, A. (2007). G*Power 3: A flexible statistical power analysis program for the social, behavioral, and biomedical sciences. Behavior Research Methods, 39, 175-191.

Gross, J. J. (2001). Emotion regulation in adulthood: Timing is everything. Current Directions in Psychological Science, 10, 214-219. 
Gross, J. J., \& John, O. P. (2003). Individual differences in two emotion regulation processes: Implications for affect, relationships, and well-being. Journal of Personality and Social Psychology, 85, 348-362.

Gross, J. J., \& Thompson, R. A. (2007). Emotion regulation: Conceptual foundations. In J. J. Gross (Ed.), Handbook of emotion regulation (pp. 3-24). New York, NY: Guilford Press.

Hemphill, J. F. (2003). Interpreting the magnitudes of correlation coefficients. American Psychologist, 58, 78-80.

Huh, T. J., Kramer, J. H., Gazzaley, A., \& Delis, D. C. (2006). Response bias and aging on a recognition memory task. Journal of the International Neuropsychological Society, 12, 17.

Jackson, D. C., Malmstadt, J. R., Larson, C. L., \& Davidson, R. J. (2000). Suppression and enhancement of emotional responses to unpleasant pictures. Psychophysiology, 37, 515522.

Koen, J. D., Aly, M., Wang, W. C., \& Yonelinas, A. P. (2013). Examining the causes of memory strength variability: Recollection, attention failure, or encoding variability?. Journal of Experimental Psychology: Learning, Memory, and Cognition, 39, 1726-1741.

Linehan, M. (1993). Cognitive-behavioral treatment of borderline personality disorder. New York, NY: Guilford press.

Lovibond, S.H. \& Lovibond, P.F. (1995). Manual for the Depression Anxiety Stress Scales. (2nd. Ed.) Sydney, Australia: Psychology Foundation.

Migo, E. M., Mayes, A. R., \& Montaldi, D. (2012). Measuring recollection and familiarity: Improving the remember/know procedure. Consciousness and Cognition, 21, 1435-1455. 
New, A. S., Fan, J., Murrough, J. W., Liu, X., Liebman, R. E., Guise, K. G., ... \& Charney, D. S. (2009). A functional magnetic resonance imaging study of deliberate emotion regulation in resilience and posttraumatic stress disorder. Biological Psychiatry, 66, 656-664.

Oswald, F. L., McAbee, S. T., Redick, T. S., \& Hambrick, D. Z. (2015). The development of a short domain-general measure of working memory capacity. Behavior Research Methods, 47, 1343-1355.

Palmer, B., Gignac, G., Bates, T., \& Stough, C. (2003). Examining the structure of the Trait Meta-Mood Scale. Australian Journal of Psychology, 55, 154-158.

Patel, R. (2009). The fear factor: Effects of implicit and explicit processing on the emotional enhancement of memory (Master's thesis). Retrieved from http://catalogue.library.ryerson.ca/record=b2037912.

Psychology Software Tools, Inc. [E-Prime 2.0]. (2012). Retrieved from http://www.pstnet.com. Qualtrics, P. (2017). UT, USA. Retrieved from http://www.qualtrics.com.

Rasch, B., Spalek, K., Buholzer, S., Luechinger, R., Boesiger, P., Papassotiropoulos, A., \& de Quervain, D. F. (2009). A genetic variation of the noradrenergic system is related to differential amygdala activation during encoding of emotional memories. PNAS, 106, $19191-19196$.

Ratcliff, R. (1978). A theory of memory retrieval. Psychological Review, 85, 59.

Richards, J. M., \& Gross, J. J. (1999). Composure at any cost? The cognitive consequences of emotion suppression. Personality and Social Psychology Bulletin, 25, 1033-1044.

Salovey, P., Mayer, J. D., Goldman, S. L., Turvey, C., \& Palfai, T. P. (1995). Emotional attention, clarity, and repair: Exploring emotional intelligence using the Trait Meta-Mood 
Scale. In J. W. Pennebaker (Ed.), Emotion, disclosure, \& health (pp. 125-154).

Washington, DC: American Psychological Association.

Schmeichel, B. J., Volokhov, R. N., \& Demaree, H. A. (2008). Working memory capacity and the self-regulation of emotional expression and experience. Journal of Personality and Social Psychology, 95, 1526-1540.

Sharot, T., \& Yonelinas, A. P. (2008). Differential time-dependent effects of emotion on recollective experience and memory for contextual information, Cognition, 106, 538-547.

Sharot, T., Verfaellie, M., \& Yonelinas, A. P. (2007) How emotion strengthens the recollective experience: A time-dependent hippocampal process. PLOS ONE 2(10): e1068.

Sheppes, G., Scheibe, S., Suri, G., Radu, P., Blechert, J., \& Gross, J. J. (2014). Emotion regulation choice: A conceptual framework and supporting evidence. Journal of Experimental Psychology: General, 143, 163-181.

Sommer, T., Gläscher, J., Moritz, S., \& Büchel, C. (2008). Emotional enhancement effect of memory: Removing the influence of cognitive factors. Learning \& Memory, 15, 569-573.

Storbeck, J., \& Clore, G. L. (2007). On the interdependence of cognition and emotion. Cognition and Emotion, 21, 1212-1237.

Tabachnick, B. G., \& Fidell, L. S. (2006). Using multivariate statistics (5th ed.). Needham Heights, MA: Allyn \& Bacon, Inc.

Tulving, E. (1985). Memory and consciousness. Canadian Psychology/Psychologie Canadienne, 26, 1-12.

Turk, D. J., Cunningham, S. J., \& Macrae, C. N. (2008). Self-memory biases in explicit and incidental encoding of trait adjectives. Consciousness and Cognition, 17, 1040-1045. 
Turner, M. L., \& Engle, R. W. (1989). Is working memory capacity task dependent?. Journal of Memory and Language, 28, 127-154.

Wixted, J. T., \& Mickes, L. (2010). A continuous dual-process model of remember/know judgments. Psychological Review, 117, 1025-1054.

Yonelinas, A. P., Kroll, N. E. A., Dobbins, I. G., \& Soltani, M. (1999). Recognition memory for faces: When familiarity supports associative recognition judgments. Psychonomic Bulletin \& Review, 6, 654-661.

Yonelinas, A. P. (2002). The nature of recollection and familiarity: A review of 30 years of research. Journal of Memory and Language, 46, 441-517. 